

\title{
The zeta function of monomial deformations of Fermat hypersurfaces
}

\author{
Remke Kloosterman
}

This paper intends to give a mathematical explanation for results on the zeta function of some families of varieties recently obtained in the context of mirror symmetry. In the process we obtain concrete and explicit examples for some results recently used in algorithms to count points on smooth hypersurfaces in $\mathbb{P}^{n}$.

In particular, we extend the monomial-motive correspondence of Kadir and Yui and we give explicit solutions to the $p$-adic Picard-Fuchs equation associated with monomial deformations of Fermat hypersurfaces.

As a byproduct we obtain Poincaré duality for the rigid cohomology of certain singular affine varieties.

\section{Introduction}

One of the families under consideration in this paper is the famous one-parameter family (Dwork family) of quintic threefolds $X_{\bar{\lambda}} \subset \mathbb{P}_{\mathbb{F}_{q}}^{4}$ given by

$$
x_{0}^{5}+x_{1}^{5}+x_{2}^{5}+x_{3}^{5}+x_{4}^{5}+\bar{\lambda} x_{0} x_{1} x_{2} x_{3} x_{4}=0,
$$

where $\bar{\lambda} \in \mathbb{F}_{q}$ is a parameter. Candelas et al. [2003] observed that the zeta function of this variety can be written as

$$
\frac{R_{1}(t, \bar{\lambda}) R_{2}(t, \bar{\lambda})^{20} R_{3}(t, \bar{\lambda})^{30}}{(1-t)(1-q t)\left(1-q^{2} t\right)\left(1-q^{3} t\right)},
$$

where the $R_{i}$ are of degree 4 . Candelas et al. gave expressions in $\bar{\lambda}$ for the zeroes of the $R_{i}$ : to explain this, note that we can lift this family to a family over the ring $\mathbb{Z}_{q}$ of Witt vector over $\mathbb{F}_{q}$. This enables us to consider this family as a family in $\mathbb{P}^{4}$ over the field of fractions $\mathbb{Q}_{q}$ of $\mathbb{Z}_{q}$. Assume that $\bar{\lambda} \in \mathbb{F}_{q}$ is chosen such that $X_{\bar{\lambda}}$ is smooth. Denote by $\lambda$ the Teichmüller lift of $\bar{\lambda}$. Specifically, Candelas et al. show

MSC2000: primary 14G10; secondary 14G15, $11 \mathrm{G} 25$.

Keywords: zeta function, $p$-adic Picard-Fuchs equation, Monsky-Washnitzer cohomology.

This work was partially supported by the DFG Schwerpunktprogramm "Globale Methoden in der komplexen Geometrie" under grant HU 337/5-3. 
that the zeroes of the zeta function of $X_{\bar{\lambda}}$ can be expressed in certain solutions of the $p$-adic Picard-Fuchs equation (associated with the family $X_{\bar{\lambda}}$ ) evaluated at $\lambda$.

This fact was proved in a more general context, but less explicitly, by N. Katz [1968]. His description of the zeta function in terms of the Picard-Fuchs equation is exploited by Lauder [2004] in order to give an algorithm to count points on smooth hypersurfaces in $\mathbb{P}^{n}$.

Some other families are investigated by Kadir [2004]. She obtained similar results. From this, one might conjecture that various factors of the zeta function are enumerated by so-called (admissible) monomial types modulo certain equivalence relations. We come back to this in Section 1.3.

Kadir and Yui [2006] noticed that monomial types are occurring in the study of several objects related to (1), for example in the Picard-Fuchs equation or in the enumeration of the factors of the zeta function. In the case $\lambda=0$, they also appear in the enumeration of the Jacobi sums needed to compute the number of points of the variety at $\lambda=0$. They proved a certain correspondence between these monomial types for Fermat varieties. Our aim is to present a different view on the above mentioned phenomena.

We should mention that N. Katz [2007] and Rojas-Leon and Wan [2007] studied the zeta function of families similar to (1) by using ( $\ell$-adic) hypergeometric sheaves. We recommend [Katz 2007] for a discussion on previous results on the Dwork family.

The main object of study in this paper are families $X_{\bar{\lambda}} / \mathbb{F}_{q}$ defined by the vanishing of polynomials of the form

$$
F_{\bar{\lambda}}:=\sum_{i=0}^{n} x_{i}^{d_{i}}+\bar{\lambda} \prod_{i} x_{i}^{a_{i}}
$$

in a weighted projective space $\mathbb{P}:=\mathbb{P}\left(w_{0}, \ldots, w_{n}\right)$, with $w_{i} d_{i}=d$ for all $i$, the $a_{i}$ are nonnegative and $\sum w_{i} a_{i}=d$; moreover, we assume that $\operatorname{gcd}(q, d)=1$. Such families will be called one-parameter monomial deformations of a Fermat hypersurface. For the rest of the introduction fix such a weighted projective space, and such a one-parameter deformation of a Fermat hypersurface. Let $\boldsymbol{a}$ denote the vector $\left(w_{0} a_{0}, w_{1} a_{1}, \ldots, w_{n} a_{n}\right) \in(\mathbb{Z} / d \mathbb{Z})^{n+1}$. We call $\boldsymbol{a}$ the deformation vector.

The main technical result of this paper implies that the $p$-adic Picard-Fuchs equation associated with such a family is a generalized hypergeometric differential equation. We refer to Sections 1.2 and 5 for more on this.

Let $U_{\bar{\lambda}}:=\mathbb{P} \backslash X_{\bar{\lambda}}$. Since

$$
Z\left(X_{\bar{\lambda}}, t\right) Z\left(U_{\bar{\lambda}}, t\right)=Z(\mathbb{P}, t),
$$

the value of $Z\left(X_{\bar{\lambda}}, t\right)$ is uniquely determined by $Z\left(U_{\bar{\lambda}}, t\right)$. Hence from now on we will only discuss how to calculate $Z\left(U_{\bar{\lambda}}, t\right)$. 
1.1. Choice of the cohomology theory. The Lefschetz fixed point formula allows us to prove statements on the zeta function by considering the action of geometric Frobenius on certain cohomology groups. Very often one uses étale cohomology. This is particularly useful when one wants to compare results in characteristic $p>0$ with results in characteristic 0 , or if one wants to consider Galois-representations on certain $\ell$-adic vector spaces.

However, for our purposes it seems more natural to use $p$-adic cohomology theories instead. One can represent cohomology classes of a variety over a finite field $\mathbb{F}_{q}$ by differential forms with coefficients in $\mathbb{Q}_{q}$. This allows us to perform several (basic) analytic tricks when computing with cohomology classes.

To be more precise, let $\lambda$ be a lift of $\bar{\lambda}$ to $\mathbb{Q}_{q}$, let $F_{\lambda}$ be a lift of $F_{\bar{\lambda}}$ and $U_{\bar{\lambda}}$. Since $U_{\lambda}$ is affine, we can define Monsky-Washnitzer groups cohomology (see Section 3) $H^{i}\left(U_{\lambda}, \mathbb{Q}_{q}\right)$. The elements in $H^{i}\left(U_{\lambda}, \mathbb{Q}_{q}\right)$ are differential forms with $\mathbb{Q}_{q}$-coefficients. There is a lift Frob ${ }_{q}$ of the Frobenius acting on these groups.

To illustrate how explicitly one can compute with Monsky-Washnitzer cohomology, we proceed to produce a basis for $H^{i}\left(U_{\lambda}\right)$. Let

$$
\Omega:=\left(\prod_{j} x_{j}\right) \sum(-1)^{i} w_{i} \frac{d x_{0}}{x_{0}} \wedge \frac{d x_{1}}{x_{1}} \wedge \cdots \wedge \frac{\widehat{d x_{i}}}{x_{i}} \wedge \cdots \wedge \frac{d x_{n}}{x_{n}} .
$$

Proposition 1.1. Let $X_{\bar{\lambda}}$ be quasismooth. Then the cohomology groups $H^{i}\left(U_{\lambda}, \mathbb{Q}_{q}\right)$ are zero except for $i=0, n$. The group $H^{0}\left(U_{\lambda}, \mathbb{Q}_{q}\right)$ is one-dimensional and Frobenius acts trivially on it. The following set is a basis for $H^{n}\left(U_{\lambda}, \mathbb{Q}_{q}\right)$ :

$$
\left\{\frac{\prod_{i=0}^{n} x_{i}^{k_{i}}}{\left(F_{\lambda}\right)^{t}} \Omega: 0 \leq k_{i}<d_{i}-1 \forall i, \sum_{i} w_{i}\left(k_{i}+1\right)=t d\right\} .
$$

This basis will be called the standard basis. We are not aware of a proper reference for this standard fact in our context. We prove this proposition in Section 3. Proposition 1.1 is a combination of Theorem 3.8 and Proposition 3.16.

The proof is based on the fact that for quasismooth $X_{\bar{\lambda}}$, de Rham cohomology of $U_{\lambda}$ with $\mathbb{Q}_{p}$ coefficients is isomorphic to the Monsky-Washnitzer cohomology of $U_{\bar{\lambda}}$ [Baldassarri and Chiarellotto 1994]. By a theorem of Steenbrink [1977] we have the isomorphism

$$
H_{\mathrm{dR}}^{n}\left(U_{\lambda}\right) \cong \bigoplus_{t>0} H^{0}\left(\Omega^{n}\left(t X_{\lambda}\right)\right) / d H^{0}\left(\Omega^{n-1}\left((t-1) X_{\lambda}\right)\right) .
$$

The vector space on the right-hand side is very well understood.

However, if $X_{\bar{\lambda}}$ is not quasismooth then the dimension of the right-hand side depends on the choice of the lift $\lambda$. If we choose $\lambda$ in such a way that $X_{\lambda}$ is not quasismooth then the right-hand side is infinite-dimensional. In that case one needs 
to add more relations to get an isomorphism with $H^{n}\left(U_{\lambda}\right)$. Which relations one needs to add is not very well understood.

A vector

$$
\boldsymbol{k}:=\left(\overline{w_{0}\left(k_{0}+1\right)}, \ldots, \overline{w_{n}\left(k_{n}+1\right)}\right) \in \prod_{i}\left(w_{i} \mathbb{Z} / d \mathbb{Z}\right)
$$

is called an admissible monomial type if for all $i$, we have $k_{i} \not \equiv-1 \bmod d_{i}$ and $\sum \overline{w_{i}\left(k_{i}+1\right)} \equiv 0 \bmod d$. Fix an admissible monomial type $\boldsymbol{k}$. Take elements $k_{i} \in \mathbb{Z}$ satisfying $0 \leq k_{i} \leq d_{i}-2$ and $k_{i} \equiv \bar{k}_{i} \bmod d_{i}$. Then with $\boldsymbol{k}$ we associate the standard basis vector

$$
\omega_{k}:=\frac{\prod x_{i}^{k_{i}}}{\left(F_{\lambda}\right)^{t}} \Omega .
$$

Remark 1.2. The results mentioned in Section 3 imply that

$$
Z\left(U_{\bar{\lambda}}, t\right)=\frac{\left(\operatorname{det}\left(I-q^{n}\left(\operatorname{Frob}_{q}^{*}\right)^{-1} t \mid H^{n}\left(U_{\lambda}, \mathbb{Q}_{q}\right)\right)\right)^{(-1)^{n+1}}}{\left(1-q^{n} t\right)} .
$$

From here on we formulate our results in terms of the characteristic polynomial of $q^{n}\left(\operatorname{Frob}_{q}^{*}\right)^{-1}$ on $H^{n}\left(U_{\lambda}, \mathbb{Q}_{q}\right)$, rather than in terms of $Z\left(U_{\bar{\lambda}}, t\right)$.

1.2. Deformation behavior. We produce a solution to the $p$-adic Picard-Fuchs equation that turns out to give us a description of the dependence of $\lambda$ of the action of Frobenius on $H^{n}\left(U_{\lambda}\right)$, where $\lambda$ is in the $p$-adic unit disc.

Following [Katz 1968], we consider the commutative diagram

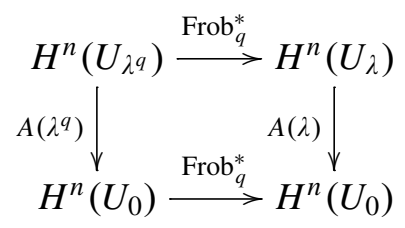

where $\lambda$ is on a small $p$-adic disc around the origin, and $A$ is a solution to the Picard-Fuchs equation associated with the family $X_{\lambda}$. Using $p$-adic analytic continuation we can extend $A(\lambda)^{-1} \operatorname{Frob}_{q, 0}^{*} A\left(\lambda^{q}\right)$ to the closed unit disc, although $A(\lambda)$ itself cannot be extended to the $p$-adic unit disc.

Let $\lambda_{0} \in \mathbb{Q}_{q}$ be the Teichmüller lift of some element $\bar{\lambda}_{0} \in \mathbb{F}_{q}$. Then $\lambda_{0}^{q}=\lambda_{0}$, hence the above diagram implies that the action of Frob $_{q}$ on $H^{n}\left(U_{\lambda_{0}}\right)$ can be recovered from the $p$-adic analytic continuation of $A(\lambda)^{-1} \operatorname{Frob}_{q, 0}^{*} A\left(\lambda^{q}\right)$. Therefore, to determine the zeta function of $X_{\bar{\lambda}_{0}}$ we need to know the Frobenius action in the Fermat-case (see 1.3) and compute the correct solution of the Picard-Fuchs equation. 
We describe the action of $A(\lambda)$ on the standard basis. We call two monomial types $\boldsymbol{k}$ and $\boldsymbol{m}$ strongly equivalent if and only if there is a $j_{0}$ such that $\boldsymbol{k}-\boldsymbol{m}=j_{0} \boldsymbol{a}$, where $\boldsymbol{a}$ is the deformation vector (see above).

Theorem 1.3. Let $\boldsymbol{k}$ be an admissible monomial type. Write $A(\lambda) \omega_{\boldsymbol{k}}=\sum c_{\boldsymbol{m}}(\lambda) \omega_{\boldsymbol{m}}$, where the sum is taken over all admissible monomial types. Then $c_{m}(\lambda)$ is nonzero only if $\boldsymbol{k}$ and $\boldsymbol{m}$ are strongly equivalent. If this is the case then $c_{\boldsymbol{m}}(\lambda)$ is of the form $c_{0} \lambda^{j_{0}} F\left(\alpha_{i} ; \beta_{j} ; \lambda^{d} c_{1}^{d}\right)$, with $F$ a p-adic generalized hypergeometric function with parameters $\alpha_{i}, \beta_{j}$ and $j_{0} \in\{0,1, \ldots, d-1\}$ is chosen such that $\boldsymbol{k}-\boldsymbol{m}=\bar{j}_{0} \boldsymbol{a}$.

Explicit formulas for the $\alpha_{i}, \beta_{j}, c_{0}$ and $c_{1}$ are given in Lemma 5.1 and Proposition 5.3. See Section 5 for a proof of Theorem 1.3.

In our proof we exploit the fact that there is a straightforward way of computing in groups like $H^{n}\left(U_{\lambda}\right)$, relying on the fact that this group is a quotient of a module of differentials over a power series ring. This allows us to perform some easy analytic operations that would be impossible in a module of differentials over a polynomial ring.

1.3. Factorization of the zeta function. We call the case $\lambda=0$ the Fermat case. One can show that Frob* on $H^{n}\left(U_{0}, \mathbb{Q}_{q}\right)$ sends the standard basis vector $\omega_{\boldsymbol{k}}$ to a constant $c_{k, q}$ times the standard basis vector $\omega_{\bar{q} k}$. Hence, if $q \equiv 1 \bmod d$ then the standard basis is a basis of eigenvectors for Frob* ${ }_{q}^{*}$. In this case Theorem 1.3 tells us that for every admissible monomial type $\boldsymbol{k}$ the operator Frob $b_{q, \lambda}$ fixes the subspace spanned by the $\omega_{\boldsymbol{m}}$, where $\boldsymbol{m}$ is strongly equivalent to $\boldsymbol{k}$.

The general case is slightly different, for this we introduce another equivalence relation: we call two monomial types $\boldsymbol{k}$ and $\boldsymbol{m}$ weakly equivalent if $j_{0} \in \mathbb{Z} / d \mathbb{Z}$ and invertible $s, t \in(\mathbb{Z} / d \mathbb{Z})^{*}$ exists such that $s \boldsymbol{k}+t \boldsymbol{m}=j_{0} \boldsymbol{a}$.

Theorem 1.4. Let $\boldsymbol{k}$ be an admissible monomial type. Write

$$
\operatorname{Frob}_{q, \lambda} \omega_{k}=\sum c_{\boldsymbol{m}}(\lambda) \omega_{\boldsymbol{m}},
$$

where the sum is taken over all admissible monomial types. Then $c_{m}(\lambda)$ is nonzero only if $\boldsymbol{k}$ and $\boldsymbol{m}$ are weakly equivalent.

This is a weak form of Theorem 6.4. Theorem 1.4 implies that the zeta function of $U_{\lambda}$ can be factored (as a rational function with $\mathbb{Q}(\lambda)$-coefficients) in such a way that each factor corresponds to a weak-equivalence class. If one only considers the zeta function over fields containing all $d$-th roots of unity, then there is a factorization of the zeta function of $U_{\lambda}$ such that each factor corresponds to a strong-equivalence class.

Explicitly determining the constants $c_{k, q}$ is actually very hard. In some cases it is known that the eigenvalues of Frob ${ }_{q}^{*}$ correspond to the Fourier coefficients of a modular form. For example if $n=2, w=(1,1,1)$ and $d=3$, then $X_{0}$ is the $j=0$ 
elliptic curve $x_{0}^{3}+x_{1}^{3}+x_{2}^{3}$. Also the case $n=3, w=(1,1,1,1), d=4$ and the case $n=5, w=(1,1,1,1), d=3$ are known to correspond to modular forms; see [Hulek and Kloosterman 2007; Shioda and Inose 1977].

A more general result on $c_{k, q}$ is due to Weil: Assume that $\mathbb{F}_{q} \supset \mathbb{F}_{p}\left(\zeta_{d}\right)$. Let $\chi$ be the $d$-th power residue symbol. Let $\boldsymbol{k}$ be an admissible monomial type. Let $\boldsymbol{k}_{i}$ be the $i$-th entry of $\boldsymbol{k}$, i.e., $w_{i}\left(k_{i}+1\right)$. Then

$$
J_{k, q}:=(-1)^{n+1} \sum_{\left(v_{1}, \ldots, v_{n}\right) \in \mathbb{E}_{q}^{n}: \sum_{i} v_{i}=-1} \chi\left(v_{1}\right)^{\boldsymbol{k}_{1}} \chi\left(v_{2}\right)^{\boldsymbol{k}_{2}} \ldots \chi\left(v_{n}\right)^{\boldsymbol{k}_{n}} .
$$

The following theorem coincides with Corollary 6.9.

Theorem 1.5. Assume $q$ is chosen such that $\mathbb{F}_{q} \supset \mathbb{F}_{p}\left(\zeta_{d}\right)$. Let $\boldsymbol{k}$ be an admissible monomial type. Let $\boldsymbol{S}$ be the set of monomial types that are weakly equivalent to $\boldsymbol{k}$. Then the sets $\left\{q^{n-1} / c_{\boldsymbol{m}, q}: m \in S\right\}$ and $\left\{J_{\boldsymbol{m}, q}: m \in S\right\}$ coincide.

1.4. Monomial-motive correspondence. We call $\boldsymbol{b} \in(\mathbb{Z} / d \mathbb{Z})^{n+1}$ an admissible automorphism type if $\boldsymbol{b}=\left(w_{0} b_{0}, w_{1} b_{1}, \ldots, w_{n} b_{n}\right) \in(\mathbb{Z} / d \mathbb{Z})^{n+1}$ is such that

$$
\sum w_{i} b_{i} a_{i} \equiv 0 \bmod d
$$

Define $\sigma_{\boldsymbol{b}}$ to be the automorphism

$$
\left[x_{0}: x_{1}: \cdots: x_{n}\right] \mapsto\left[\zeta_{d}^{w_{0} b_{0}} x_{0}: \zeta_{d}^{w_{1} b_{1}} x_{1}: \cdots: \zeta_{d}^{w_{n} b_{n}} x_{n}\right] .
$$

We call two monomial types $\boldsymbol{k}$ and $\boldsymbol{m}$ distinguishable by automorphisms if there exists an admissible automorphism type $\boldsymbol{b} \in(\mathbb{Z} / d \mathbb{Z})^{n+1}$ such that

$$
\sigma_{\boldsymbol{b}}\left(\prod x_{i}^{k_{i}}\right)=\prod x_{i}^{k_{i}} \quad \text { and } \quad \sigma_{\boldsymbol{b}}\left(\prod x_{i}^{m_{i}}\right) \neq \prod x_{i}^{m_{i}} .
$$

Theorem 1.6. Two monomial types $\boldsymbol{k}$ and $\boldsymbol{m}$ are weakly equivalent if and only if $\boldsymbol{k}$ and $\boldsymbol{m}$ are not distinguishable by automorphisms.

This result enables us to give a different proof for the monomial-motive correspondence of Kadir and Yui [2006], and to generalize it as follows: fix an admissible monomial type $\boldsymbol{k}$. Let $G_{\boldsymbol{k}}$ be the group of automorphisms of the form $\sigma_{\boldsymbol{b}}$ that fix $\omega_{\boldsymbol{k}}$. Then the subspace of $H^{n}(U)$ fixed by $G_{\boldsymbol{k}}$ is the spanned by the $\omega_{\boldsymbol{m}}$ such that $\boldsymbol{m}$ is weakly equivalent to $\boldsymbol{k}$. This can be also extended to the level of motives, i.e., we find a submotive $\mathfrak{h}\left(U_{\lambda} / G_{\boldsymbol{k}}\right)$ of the (Chow-)motive $\mathfrak{h}\left(U_{\lambda}\right)$. Moreover, we obtain that

$$
\mathfrak{h}\left(U_{\lambda}\right)=\bigoplus_{[k]} \mathfrak{h}\left(U_{\lambda} / G_{k}\right)
$$

where we sum over all the weak-equivalence classes.

Kadir and Yui decompose $\mathfrak{h}\left(U_{\lambda} / G_{\boldsymbol{k}}\right)$ further. To explain this, we need to change our context, and consider our family $X_{\lambda}$ over the field $\mathbb{Q}$ of rational numbers. Then 
the Galois group $\operatorname{Gal}\left(\mathbb{Q}\left(\zeta_{d}\right) / \mathbb{Q}\right)$ acts nontrivially on $G_{k}$, and this enables us to find correspondences in $C H^{n}\left(U_{\lambda} / G_{\boldsymbol{k}} \times U_{\lambda} / G_{\boldsymbol{k}}\right)$ that decompose $\mathfrak{h}\left(U_{\lambda} / G_{\boldsymbol{k}}\right)$ into smaller motives. It is easy to see that each such motive corresponds to a strongequivalence class of monomial types. This correspondence between admissible monomial types and submotives of $\mathfrak{h}^{n}\left(U_{\lambda}\right)$ is called by Kadir and Yui monomialmotive correspondence. They also relate monomial types with the Picard-Fuchs equation. For this issue we refer to Section 1.2.

Kadir and Yui [2006] could only prove their monomial-motive correspondence if $X_{\lambda}$ is a Calabi-Yau hypersurface of dimension 3 and $\lambda=0$. The above discussion extends this correspondence to any quasismooth member of a one-parameter monomial deformation of a Fermat hypersurface in a weighted projective space, for any degree $d$ such that $w_{i} \mid d$ for all $i$ and provided that the characteristic does not divide $d$.

Kadir and Yui prove the monomial-motive correspondence using Jacobi sums. We take a more direct approach using subgroups of the automorphism group.

This paper is organized as follows: in Section 2 we fix some notation and list some standard definitions. In Section 3 we discuss Monsky-Washnitzer cohomology groups and recall some of the properties of these groups. In Section 4 we recall Katz' result on the deformation of the zeta function of a hypersurface in $\mathbb{P}^{n}$. In Section 5 we make Katz' result explicit. In Section 6 we discuss the Frobenius action on the cohomology of a Fermat hypersurface and prove some results on the structure of the zeta function of a monomial deformation of a Fermat hypersurface.

\section{Notation}

Fix once and for all :

- a prime $p$ (the characteristic) and a positive integer $r$,

- an integer $n$ (the dimension of the ambient space),

- a vector $\left(w_{0}, w_{1}, \ldots, w_{n}\right) \in \mathbb{Z}^{n+1}$ such that none of the $w_{i}$ is divisible by $p$.

- an integer $d$ divisible by all the $w_{i}$ and $p$ does not divide $d$.

Set $q=p^{r}$ and $d_{i}:=d / w_{i}$. Let $\mathbb{Q}_{q}$ denote the unique unramified extension of degree $r$ of $\mathbb{Q}_{p}$. Let $w:=\sum w_{i}$ denote the total weight. Let $\mathbb{P}_{\mathbb{F}_{q}}:=\mathbb{P}_{\mathbb{F}_{q}}^{n}\left(w_{0}, \ldots, w_{n}\right)$ be the associated weighted projective space over the finite field $\mathbb{F}_{q}$.

Definition 2.1. A monomial type $\boldsymbol{m}=\left(\bar{m}_{0}, \ldots, \bar{m}_{n}\right)$ is an element of $\prod_{i} w_{i} \mathbb{Z} / d \mathbb{Z}$ such that $\sum \bar{m}_{i}=0$ in $\mathbb{Z} / d \mathbb{Z}$. Choose representatives $m_{i} \in \mathbb{Z}$ of $\bar{m}_{i}$ such that $0 \leq m_{i}<d$. The relative degree of $\boldsymbol{m}$ is $\sum m_{i} / d$.

Fix once and for all a monomial type $\boldsymbol{a}$ of relative degree 1, with at least 2 nonzero entries. We call $\boldsymbol{a}$ the deformation vector. Let $a_{i}$ be integers such that 
$0 \leq a_{i}<d_{i}$ and $\boldsymbol{a} \equiv\left(\bar{w}_{0} \bar{a}_{0}, \ldots, \bar{w}_{n} \bar{a}_{n}\right)$. Set

$$
F_{\lambda}:=\sum x_{i}^{d_{i}}+\lambda \prod x_{j}^{a_{j}} .
$$

Let $F:=F_{0}$. If $\bar{\lambda} \in \mathbb{F}_{q}$, denote by $X_{\bar{\lambda}}$ the zero set of $F_{\bar{\lambda}}$ in $\mathbb{P}$. If $\lambda \in \mathbb{Q}_{q}$, denote by $X_{\lambda}$ the zero set of $F_{\lambda}$. Let $U_{\bar{\lambda}}$ be the complement $\mathbb{P} \backslash X_{\bar{\lambda}}$. Let $U_{\lambda}$ be the complement $\mathbb{P} \backslash X_{\lambda}$.

$$
\text { Let } \Omega:=\prod_{i} x_{i} \sum_{j}(-1)^{j} w_{j} \frac{d x_{0}}{x_{0}} \wedge \cdots \wedge \frac{\widehat{d x_{j}}}{x_{j}} \wedge \frac{d x_{n}}{x_{n}} \text {. }
$$

Definition 2.2. A monomial type $\boldsymbol{k}$ is called admissible if there exist integers $k_{i}$, for $i=0, \ldots, n$, such that $0 \leq k_{i} \leq d_{i}-2$ and $\boldsymbol{k}=\left(w_{0}\left(k_{0}+1\right), \ldots, w_{n}\left(k_{n}+1\right)\right)$. Let $t$ be the relative degree of $\boldsymbol{k}$. With $\boldsymbol{k}$ we associate the differential form

$$
\omega_{\boldsymbol{k}}:=\frac{\prod x_{i}^{k_{i}}}{F_{\lambda}^{t}} \Omega \text {. }
$$

Denote by $(a)_{m}$ the Pochhammer symbol $a(a+1) \ldots(a+m-1)$.

Definition 2.3. Let $\pi: \mathbb{P}^{n} \rightarrow \mathbb{P}$ be the natural quotient map sending $x_{i}$ to $x_{i}^{w_{i}}$. Let $G:=\times \boldsymbol{\mu}_{w_{i}} / \Delta$ be the Galois group associated with this quotient. We call $\pi$ the standard quotient map and $G$ the group associated with $\pi$.

\section{Monsky-Washnitzer cohomology}

We will not define rigid cohomology in complete detail, but give a simplified presentation for the case of quasismooth hypersurfaces. For a good introduction to the theory of rigid cohomology we refer to [Berthelot 1983; 1997b].

Since $U_{\lambda}$ is affine, we can write $U_{\lambda}=\operatorname{Spec} R_{\lambda}$, with

$$
R_{\lambda}=\mathbb{Q}_{q}\left[\lambda, Y_{0}, \ldots, Y_{m}\right] /\left(G_{1, \lambda}, \ldots, G_{k, \lambda}\right) .
$$

Definition 3.1. Fix $\lambda_{0}$ in the closed $p$-adic unit disc and set

$$
R_{\lambda_{0}}^{\dagger}=\frac{\left\{H \in \mathbb{Q}_{q} \llbracket Y_{0}, \ldots, Y_{m} \rrbracket: \text { the radius of convergence of } H \text { is at least } r>1\right\}}{\left(G_{1, \lambda_{0}}, \ldots, G_{k, \lambda_{0}}\right)} \text {. }
$$

Then $R_{\lambda_{0}}^{\dagger}$ is called the overconvergent completion (or weak completion) of $R_{\lambda_{0}}$.

If $\pi$ is the standard quotient map, $G$ is its associated group (Definition 2.3), and $S:=S_{\lambda_{0}}^{\dagger}$ is the overconvergent completion of the coordinate ring of $\mathbb{P}^{n} \backslash \pi^{-1}\left(X_{\lambda_{0}}\right)$, there is on the module of differential forms $\Omega_{S}^{i}$ a natural $G$-action. Set $\Omega_{R}^{i}=$ $\left(\Omega_{S}^{i}\right)^{G}$. The $i$-th Monsky-Washnitzer cohomology group $H^{i}\left(U_{\lambda_{0}}, \mathbb{Q}_{q}\right)$ is the $i$-th cohomology group of the complex $\Omega_{R}^{\bullet}$.

Notation 3.2. Let $X \subset \mathbb{P}$ be a quasiprojective variety. Denote by $H_{\text {rig }}^{i}(X)$ the $i$-th rigid cohomology group of $X$ and by $H_{\mathrm{rig}, c}^{i}(X)$ the $i$-th rigid cohomology group with compact support of $X$, as defined in [Berthelot 1983]. 
There exists a second, equivalent, definition of $H^{i}\left(U_{\lambda_{0}}, \mathbb{Q}_{q}\right)$. This goes as follows: since $U_{\lambda_{0} \text {, sing }}$ is affine, there is a ring $S$ such that $U_{\lambda_{0} \text {, sing }}=\operatorname{Spec} S$. Let $S^{\dagger}$ be an overconvergent completion of $S$. Let $\imath$ : Spec $R_{\lambda_{0}}^{\dagger} \backslash \operatorname{Spec} S^{\dagger} \rightarrow \operatorname{Spec} R_{\lambda_{0}}^{\dagger}$ be the inclusion. Let $\Omega_{\text {Spec } R_{\lambda_{0}}^{\dagger}}^{i}$ be the sheaf $l_{*} \Omega_{\text {Spec } R_{\lambda_{0}}^{\dagger} \backslash \operatorname{Spec} S^{\dagger}}$. Then define the MonskyWashnitzer cohomology groups $H^{i}\left(U_{\lambda_{0}}, \mathbb{Q}_{q}\right)$ as the cohomology groups of the complex obtained by taking global sections. The proof that these two definitions are equivalent is very similar to [Dolgachev 1982, 2.2.4].

Definition 3.3. Let $R$ be a ring over $\mathbb{Z}_{q}$. Let $\pi$ be the maximal ideal of $\mathbb{Z}_{q}$. A lift of Frobenius is a ring homomorphism Frob ${ }_{q}^{*}: R \rightarrow R$ whose reduction modulo $\pi$,

$$
\operatorname{Frob}_{q}^{*} \bmod \pi: R \otimes_{\mathbb{Z}_{q}} \mathbb{F}_{q} \rightarrow R \otimes_{\mathbb{Z}_{q}} \mathbb{F}_{q},
$$

is well-defined and equals $x \mapsto x^{q}$.

Fix a lift of Frobenius $\operatorname{Frob}_{q}^{*}$ to $R_{\lambda_{0}}^{\dagger}$, such that $\operatorname{Frob}_{q}^{*}(\lambda)=\lambda^{q}$. By abuse of notation we denote by Frob* ${ }_{q}^{*}$ also the induced morphism on $H^{i}\left(U_{\lambda_{0}}, \mathbb{Q}_{q}\right)$.

Proposition 3.4. There is a natural isomorphism

$$
H_{\text {rig }}^{i}\left(U_{\bar{\lambda}_{0}}, \mathbb{Q}_{q}\right) \cong H^{i}\left(U_{\lambda_{0}}, \mathbb{Q}_{q}\right)
$$

which is compatible with the action of Frobenius.

Proof. Similar to the proof of [Berthelot 1997b, Proposition 1.10].

Definition 3.5. Let $K$ be a field. Let $G \in K\left[x_{0}, \ldots, x_{n}\right]$ be a weighted homogeneous polynomial (with weights $\left(w_{0}, \ldots, w_{n}\right)$ ). Let $Y$ be the hypersurface $G=0$ in $\mathbb{P}$. Then $Y$ is said to be quasismooth if the affine cone $\operatorname{Spec} K\left[X_{0}, \ldots, X_{n}\right] / G$ is smooth or has exactly one singular point, namely $(0,0, \ldots, 0)$.

Remark 3.6. If $\mathbb{P}=\mathbb{P}^{n}$ then a hypersurface $X \subset \mathbb{P}$ is quasismooth if and only if it is smooth.

An easy calculation shows:

Lemma 3.7. Let $I=\left\{i \in\{0,1, \ldots, n\}: a_{i} \not \equiv 0 \bmod p\right\}$. Let $g=\operatorname{gcd}_{i \in I}\left(a_{i} w_{i}\right)$ and $d^{\prime}:=d / g$. If there is a nonzero $a_{i}$ such that $a_{i} \equiv 0 \bmod p$ then $X_{\lambda}$ is quasismooth for all $\lambda$. Otherwise, $X_{\bar{\lambda}}$ is quasismooth if and only if

$$
\bar{\lambda}^{d^{\prime}} \neq \frac{(-1)^{d^{\prime}} \bar{d}^{d^{\prime}}}{\prod_{i \in I}\left(\bar{a}_{i} \bar{w}_{i}\right)^{a_{i} w_{i} / g}} .
$$

Proof. Consider the partial derivative of $F$ with respect to $x_{j}$. If $\bar{a}_{j}=0$ then this derivative equals $x_{j}^{d_{j}-1}$ and vanishes if and only if $x_{j}=0$. 
Suppose there is a $j$ such that $a_{j} \neq 0$ and $x_{j}=0$. Then for all for all $k \neq j$ we have

$$
0=\frac{\partial F_{\bar{\lambda}}}{\partial x_{k}}=\bar{d}_{k} x_{k}^{d_{k}-1}+\bar{a}_{k} \bar{\lambda} \frac{\prod x_{i}^{a_{i}}}{x_{k}}=\bar{d}_{k} x_{k}^{d_{k}-1} .
$$

This implies that all the $x_{k}$ would vanish. Hence if $X_{\bar{\lambda}}$ is singular at $\left(x_{0}: \cdots: x_{n}\right)$ then $x_{j}=0$ if $\bar{a}_{j}=0$ and $x_{j} \neq 0$ if $a_{j} \neq 0$. If there is a $j$ such that $p$ divides a nonzero $a_{j}$ then $X_{\bar{\lambda}}$ is quasismooth.

Suppose now that $p$ does not divide any of the positive $a_{i}$.

Suppose $\bar{a}_{j} \neq 0$. Consider now the derivative with respect to $x_{j}$ :

$$
\frac{\partial F_{\bar{\lambda}}}{\partial x_{j}}=\bar{d}_{j} x_{j}^{d_{j}-1}+\overline{a_{j} \lambda} \frac{\prod x_{i}^{a_{i}}}{x_{j}} .
$$

This derivative vanishes if and only if

$$
\bar{\lambda} \prod x_{i}^{a_{i}}=-\frac{\bar{d}_{j}}{\bar{a}_{j}} x_{j}^{d_{j}} .
$$

In particular,

$$
-\frac{\bar{d}_{j}}{\bar{a}_{j}} x_{j}^{d_{j}}=-\frac{\bar{d}_{k}}{\bar{a}_{k}} x_{k}^{d_{k}} \quad \text { for } j, k \in I .
$$

Fix $d_{j}$-th roots $\alpha_{j}$ of $\bar{d}_{j} / \bar{a}_{j}$. Let $\zeta$ be a primitive $d^{\prime}$-th root of unity. A solution of the above set of equations is of the form

$$
x_{j}=\frac{\gamma^{w_{j}}}{\alpha_{j}} \zeta^{k_{j} w_{j}} \quad \text { for some } \gamma, k_{j}
$$

Substituting gives

$$
\bar{\lambda} \prod_{i \in I} \frac{\zeta^{k_{i} w_{i} a_{i}}}{\alpha^{a_{i}}}=-1
$$

which is equivalent with

$$
\bar{\lambda}^{d^{\prime}}=(-1)^{d^{\prime}} \prod \alpha^{a_{j} d^{\prime}}=\frac{(-1)^{d^{\prime}} \bar{d}^{d^{\prime}}}{\prod_{i \in I}\left(\overline{a_{i} w_{i}}\right)^{a_{i} w_{i} / g}} .
$$

Let $X \subset \mathbb{P}$ be a hypersurface. Let $U=\mathbb{P} \backslash X$. Recall that we have a Gysin-type exact sequence (see [Berthelot 1983, Section 3])

$$
\cdots \rightarrow H_{\text {rig }, c}^{i-1}\left(X, \mathbb{Q}_{q}\right) \rightarrow H_{\text {rig }, c}^{i}\left(U, \mathbb{Q}_{q}\right) \rightarrow H_{\text {rig }, c}^{i}\left(\mathbb{P}, \mathbb{Q}_{q}\right) \rightarrow H_{\text {rig }, c}^{i}\left(X, \mathbb{Q}_{q}\right) \rightarrow \cdots
$$

Theorem 3.8. Let $\lambda_{0} \in \mathbb{Z}_{q}$ be such that $X_{\bar{\lambda}_{0}}$ is quasismooth. Then the groups $H^{i}\left(U_{\lambda_{0}}, \mathbb{Q}_{q}\right)$ are zero except for $i=0, n$. 
Proof. Set $X=X_{\lambda_{0}}$ and $U=U_{\lambda_{0}}$. Consider first the case $\mathbb{P}=\mathbb{P}^{n}$. From Remark 3.6 it follows that $U$ and $X$ are smooth. Since $\Omega_{R}^{i}=0$ for $i>n$ we have $H^{i}\left(U, \mathbb{Q}_{q}\right)=0$ for $i>n$. Proposition 3.4 implies that $H_{\text {rig }}^{i}\left(U, \mathbb{Q}_{q}\right)$ is trivial for $i>n$. Using Poincaré duality [Berthelot 1997a] it follows that

$$
H_{\text {rig }, c}^{i}\left(U, \mathbb{Q}_{q}\right)=0 \text { for } i<n .
$$

From (3) it follows that

$$
H_{\text {rig }, c}^{i}\left(X, \mathbb{Q}_{q}\right) \cong H_{\text {rig }, c}^{i}\left(\mathbb{P}, \mathbb{Q}_{q}\right) \text { for } i<n-1 .
$$

Using Poincaré duality, it follows that

$$
H_{\text {rig }}^{i}\left(X, \mathbb{Q}_{q}\right) \cong H_{\text {rig }}^{i}\left(\mathbb{P}, \mathbb{Q}_{q}\right) \text { for } n-1<i<2 n .
$$

Using that $X$ is compact, it follows that

$$
H_{\text {rig }, c}^{i}\left(X, \mathbb{Q}_{q}\right)=H_{\text {rig }}^{i}\left(X, \mathbb{Q}_{q}\right) \cong H_{\text {rig }}^{i}\left(\mathbb{P}, \mathbb{Q}_{q}\right)=H_{\text {rig }, c}^{i}\left(\mathbb{P}, \mathbb{Q}_{q}\right) \text { for } n-1<i<2 n .
$$

Using the sequence (3) again, we obtain that $H_{\text {rig,c }}^{i}\left(U, \mathbb{Q}_{q}\right)=0$ for $i \notin\{n, 2 n\}$. Applying Poincaré duality yields $H_{\text {rig }}^{i}\left(U, \mathbb{Q}_{q}\right)=H^{i}\left(U, \mathbb{Q}_{q}\right)=0$ for $i \notin\{0, \operatorname{dim} U\}$.

The general case can be deduced from this as follows: consider the standard quotient map $\pi: \mathbb{P}^{n} \rightarrow \mathbb{P}$ sending $x_{i}$ to $x_{i}^{w_{i}}$. Let $Y$ be $\pi^{-1}(X)$. Let $G$ be the group associated with $\pi$. From Lemma 3.7 it follows that $X$ is quasismooth if and only if $Y$ is smooth. Let $V$ be the complement of $Y$ in $\mathbb{P}^{n}$. Then from the above it follows that $H^{i}(V)=0$ except for $i=0, n$. In particular, $d \Omega_{V}^{j-1, \dagger}=\Omega_{V}^{j, \dagger}$. One easily shows that $\left(d \Omega_{V}^{j-1, \dagger}\right)^{G}=d\left(\left(\Omega_{V}^{j-1, \dagger}\right)^{G}\right)$. This implies that

$$
H^{j}(U)=\frac{\left(\Omega_{V}^{j, \dagger, c l}\right)^{G}}{\left(d \Omega_{V}^{j-1, \dagger}\right)^{G}}=0 \quad \text { if } j \neq 0, n .
$$

Remark 3.9. One might try to prove the vanishing of $H^{i}(U)$ for the complement of an arbitrary quasismooth hypersurface along the lines of the above proof. This fails if the following happens: Let $H_{1}, \ldots, H_{j}$ be the coordinate hyperplanes corresponding to coordinates with weight $w_{i}>1$. Suppose there is a subset of $\left\{H_{i}\right\}$ such that $X \cap H_{i_{1}} \cap H_{i_{2}} \cap \cdots \cap H_{i_{k}}$ is not quasismooth. Then $\pi^{-1}(X)$ is singular, so the strategy of the above proof does not apply. Conversely, if $\pi^{-1}(X)$ is singular then such a set of coordinate hyperplanes exists.

Theorem 3.10 (Poincaré duality for $H^{i}\left(U_{\lambda}, \mathbb{Q}_{q}\right)$ ). Let $\bar{\lambda}_{0} \in \mathbb{F}_{q}$ be such that $X_{\bar{\lambda}_{0}}$ is quasismooth. There is a nondegenerate pairing

$$
H_{\text {rig }, c}^{i}\left(U_{\bar{\lambda}_{0}}, \mathbb{Q}_{q}\right) \times H_{\text {rig }}^{2 n-i}\left(U_{\bar{\lambda}_{0}}, \mathbb{Q}_{q}\right) \rightarrow H_{\text {rig }, c}^{2 n}\left(U_{\bar{\lambda}_{0}}, \mathbb{Q}_{q}\right)
$$

respecting the Frobenius action. 
Proof. Set $X=X_{\lambda_{0}}$ and $U=U_{\lambda_{0}}$. Consider first the case $\mathbb{P}=\mathbb{P}^{n}$. Then from Lemma 3.7 it follows that $U$ and $X$ are smooth. The main theorem of [Berthelot 1997a] asserts the existence of such pairings.

The general case can be obtained as follows: consider the standard quotient map $\pi: \mathbb{P}^{n} \rightarrow \mathbb{P}$ sending $x_{i}$ to $x_{i}^{w_{i}}$. Let $Y$ be $\pi^{-1}(X)$. Let $G$ be the group associated with $\pi$. From Lemma 3.7 it follows that $X$ is quasismooth if and only if $Y$ is smooth. Let $V$ be the complement of $Y$ in $\mathbb{P}^{n}$. Since Poincaré duality is $G$-equivariant, one obtains a pairing

$$
H_{\text {rig }}^{i}\left(V, \mathbb{Q}_{q}\right)^{G} \times H_{\text {rig }, c}^{2 n-i}\left(V, \mathbb{Q}_{q}\right)^{G} \rightarrow H_{\text {rig, }, c}^{2 n}\left(V, \mathbb{Q}_{q}\right) .
$$

Using the isomorphism $\left(\Omega_{V}^{k}\right)^{G} \cong \Omega_{U}^{k}$, we obtain isomorphisms

$$
H_{\mathrm{rig}}^{i}\left(V, \mathbb{Q}_{q}\right)^{G} \cong H_{\mathrm{rig}}^{i}\left(U, \mathbb{Q}_{q}\right) \quad \text { and } \quad H_{\mathrm{rig}, c}^{2 n-i}\left(V, \mathbb{Q}_{q}\right)^{G} \cong H_{\mathrm{rig}, c}^{2 n-i}\left(U, \mathbb{Q}_{q}\right) \text {. }
$$

This yields the proof.

Theorem 3.11 (Lefschetz trace formula). Let $\bar{\lambda}_{0} \in \mathbb{F}_{q}$ be such that $X_{\bar{\lambda}_{0}}$ is quasismooth. Then

$$
\sum_{i}(-1)^{i} \operatorname{trace}\left(\left(q^{n}\left(\text { Frob }^{*}\right)^{-1}\right) \mid H^{i}\left(U_{\lambda_{0}}\right)\right)=\# U_{\bar{\lambda}_{0}}\left(\mathbb{F}_{q}\right) .
$$

Proof. Combine the Lefschetz trace formula for rigid cohomology with compact support [Étesse and Le Stum 1993, théorème I] with Poincaré duality (Theorem 3.10) and Proposition 3.4.

Proposition 3.12. The group $H^{0}\left(U_{\lambda}, \mathbb{Q}_{q}\right)$ is one-dimensional, and Frobenius acts trivially on $H^{0}\left(U_{\lambda}, \mathbb{Q}_{q}\right)$.

Proof. Straightforward.

Let $H_{\mathrm{dR}}^{n}\left(U_{\lambda}, \mathbb{Q}_{q}\right)$ denote the algebraic de Rham cohomology of $U_{\lambda}$.

Theorem 3.13 (Baldassarri and Chiarellotto). Suppose $\lambda$ is chosen such that $X_{\bar{\lambda}}$ is quasismooth. Then the natural map

$$
H_{\mathrm{dR}}^{n}\left(U_{\lambda}, \mathbb{Q}_{q}\right) \rightarrow H^{n}\left(U_{\lambda}, \mathbb{Q}_{q}\right)
$$

is an isomorphism.

Proof. Consider first the case $\mathbb{P}=\mathbb{P}^{n}$. Then this is precisely the main theorem of [Baldassarri and Chiarellotto 1994].

The general case can be obtained as follows: consider the standard quotient map $\pi: \mathbb{P}^{n} \rightarrow \mathbb{P}$ sending $x_{i}$ to $x_{i}^{w_{i}}$. Let $Y_{\lambda}$ be $\pi^{-1}\left(X_{\lambda}\right)$. Let $G$ be the group associated with $\pi$. From Lemma 3.7 it follows that $X_{\lambda}$ is quasismooth if and only if $Y_{\lambda}$ is smooth. Let $V_{\lambda}$ be the complement of $Y_{\lambda}$ in $\mathbb{P}^{n}$. Then we have an isomorphism

$$
H_{\mathrm{dR}}^{n}\left(V_{\lambda}, \mathbb{Q}_{q}\right) \rightarrow H^{n}\left(V_{\lambda}, \mathbb{Q}_{q}\right) .
$$


There is a natural $G$-action on both groups and it is easy to see that this isomorphism is $G$-equivariant. Moreover, using [Dolgachev 1982, Lemma 2.2.2] we obtain that $\pi$ induces isomorphisms $H_{\mathrm{dR}}^{n}\left(V_{\lambda}\right)^{G} \cong H_{\mathrm{dR}}^{n}\left(U_{\lambda}\right)$ and $H^{n}\left(V_{\lambda}\right)^{G} \cong H^{n}\left(U_{\lambda}\right)$; hence the natural map

$$
H_{\mathrm{dR}}^{n}\left(U_{\lambda}, \mathbb{Q}_{q}\right) \rightarrow H^{n}\left(U_{\lambda}, \mathbb{Q}_{q}\right)
$$

is an isomorphism.

Let $G$ be the defining equation of a quasismooth hypersurface $Y \subset \mathbb{P}$. Let $V:=\mathbb{P} \backslash Y$. Similar to the case of ordinary projective space, the algebraic de Rham cohomology of $V$ can be computed using the complex $C_{k}^{p}=\Omega^{p}((k+p) Y)$. I.e., the hypercohomology group $\mathbb{H}^{n}\left(\mathbb{P}, C_{k}^{\bullet}\right)$ equals $H^{0}\left(\mathbb{P}, C_{k}^{n}\right) / d H^{0}\left(\mathbb{P}, C_{k}^{n-1}\right)$ and

$$
H_{\mathrm{dR}}^{n}(V)=\bigoplus_{k} H^{0}\left(\mathbb{P}, C_{k}^{n}\right) / d H^{0}\left(\mathbb{P}, C_{k}^{n-1}\right) .
$$

(A proof of this equality can be obtained as follows. After fixing an embedding $\mathbb{Q}_{q} \hookrightarrow \mathbb{C}$ and tensoring both sides with $\mathbb{C}$, we obtain that it suffices to prove this result over $\mathbb{C}$. This is precisely the main result of [Steenbrink 1977].)

More explicitly, the vector space $H^{n}\left(V, \mathbb{Q}_{q}\right)$ can be identified with the quotient of the infinite-dimensional vector space spanned by

$$
\frac{H}{G^{t}} \Omega
$$

with $\operatorname{deg}(H)=t \operatorname{deg}(G)-\sum w_{i}$, by the relations

$$
\frac{(t-1) H G_{x}-G H_{x}}{G^{t}} \Omega,
$$

where the subscript $x$ means the partial derivative with respect to a coordinate $x$ on $\mathbb{P}$.

If $G=F$ (the polynomial whose zero-set is the Fermat hypersurface) then this formula reads as

$$
\frac{(t-1) d_{i} H x_{i}^{d_{i}-1}}{F^{t}} \Omega=\frac{H_{x_{i}}}{F^{t-1}} \Omega
$$

in $H^{n}(U)$. This motivates the following definition:

Definition 3.14. Let $\omega \in \Omega^{n}\left(U_{0}\right)$ be a form of the type

$$
\frac{H}{F^{t}} \Omega
$$


with $H$ a monomial. Let $x_{i}$ be a coordinate of $\mathbb{P}$ such that $x_{i}^{d_{i}-1}$ divides $H$. Then the reduction of $\omega$ with respect to $x_{i}$ is the form

$$
\frac{\frac{\partial}{\partial x_{i}}\left(\frac{H}{x_{i}^{d_{i}-1}}\right)}{(t-1) d_{i} F^{t-1}} \Omega .
$$

The complete reduction $\operatorname{red} \omega:=H^{\prime} / F^{s} \Omega$ of $\omega$ is the form obtained by successively reducing with respect to the coordinates $x_{i}$ of $\mathbb{P}$, such that for all $i$ the exponent of $x_{i}$ in $H^{\prime}$ is at most $d_{i}-2$.

Note that $\omega$ and the reduction with respect to $x_{i}$ of $\omega$ represent the same class in $H^{n}\left(U_{0}, \mathbb{Q}_{q}\right)$, and that the complete reduction of $\omega$ cannot be further reduced.

Definition 3.15. Let $P^{\bullet}$ be the pole order filtration on $H^{n}\left(U_{\lambda}\right)$, that is $\omega \in P^{t}$ if $\omega=\frac{G}{F_{\lambda}^{t}} \Omega$ for some $G \in \mathbb{Q}_{q}\left[x_{0}, \ldots x_{n}\right]$.

Let $\boldsymbol{k}$ be an admissible monomial type. Recall that we can associate a differential form $\omega_{\boldsymbol{k}}$ with it. By definition $\omega_{\boldsymbol{k}}$ lies in $P^{t}$, where $t$ is the relative degree of $\boldsymbol{k}$.

Proposition 3.16. Let $\lambda$ be such that $X_{\lambda}$ is quasismooth. Then the set

$$
\left\{\omega_{\boldsymbol{k}}: \boldsymbol{k} \text { an admissible monomial type }\right\}
$$

is a basis for $H^{n}\left(U_{\lambda}, \mathbb{Q}_{q}\right)$.

Proof. The above discussion implies the statement for $\lambda=0$.

We start by proving that for every integer $t$ the set

$$
\left\{w_{\boldsymbol{k}}: \boldsymbol{k} \text { an admissible monomial type of relative degree } t\right\}
$$

is linearly independent in $P^{t} / P^{t-1}$.

The relations in $P^{t} / P^{t-1}$ are generated by (cf. the discussion before Definition 3.14)

$$
\frac{x_{i}^{d_{i}-1} \prod x_{j}^{k_{j}}}{F_{\lambda}^{t}} \Omega=\frac{-\lambda a_{i}}{d_{i}} \frac{\prod x_{j}^{k_{j}+a_{j}}}{x_{i} F_{\lambda}^{t}} \Omega .
$$

Suppose $i$ is chosen such that $a_{i} \neq 0$. Let

$$
\sigma_{i}(G):=\frac{-d_{i} x_{i}^{d_{i}}}{\lambda a_{i} \prod_{j} x_{j}^{a_{j}}} G .
$$

If $G$ is a monomial of degree $t d-\sum w_{i}$ such that all the exponents of the $x_{j}$ are at least $a_{j}-\delta_{i, j}$, then

$$
\frac{G}{F_{\lambda}^{t}} \Omega \equiv \frac{\sigma_{i}(G)}{F_{\lambda}^{t}} \Omega \bmod P^{t-1}
$$


Note that $\sigma_{i}$ is defined if the exponent of $x_{j}$ is at least $a_{j}-\delta_{i, j} d_{i}$, but $\sigma_{i}$ corresponds to a relation in $P^{t}$ only if the exponent $x_{j}$ is at least $a_{j}-\delta_{i, j}$. Similarly, if the exponent of $x_{i}$ in $G$ is at least $d_{i}-1$, then

$$
\frac{G}{F_{\lambda}^{t}} \Omega \equiv \frac{\sigma_{i}^{-1}(G)}{F_{\lambda}^{t}} \Omega \bmod P^{t-1} .
$$

Take a nontrivial expression $\sum b_{\boldsymbol{k}} \omega_{\boldsymbol{k}}$ that is zero modulo $P^{t-1}$. Since the $\sigma_{i}$ generate the relations, and the $\sigma_{i}$ map monomials to monomials, there exists two distinct admissible monomial types $\boldsymbol{k}, \boldsymbol{m}$ of relative degree $t$ and a sequence of $\sigma_{i}$ and $\sigma_{j}^{-1}$ such that

$$
\tau\left(\omega_{k}\right):=\sigma_{i_{s}}^{\epsilon_{s}} \ldots \sigma_{i_{1}}^{\epsilon_{1}}\left(\omega_{k}\right)=c_{0} \omega_{m},
$$

with

- $c_{0} \in \mathbb{Q}_{q}$,

- $\epsilon_{j} \in\{ \pm 1\}$,

- $a_{i_{j}} \neq 0$ for all $j$,

- for all $j$ such that $\epsilon_{j}=1$ and for all $k$, the exponent of $x_{k}$ in $\sigma_{i_{j-1}}^{\epsilon_{j-1}} \ldots \sigma_{i_{1}}^{\epsilon_{1}}\left(\omega_{\boldsymbol{k}}\right)$ is at least $a_{k}-\delta_{i_{j}, k}$, and

- for all $j$ such that $\epsilon_{j}=-1$, the exponent of $x_{i_{j}}$ in $\sigma_{i_{j-1}}^{\epsilon_{j-1}} \ldots \sigma_{i_{1}}^{\epsilon_{1}}\left(\omega_{k}\right)$ is at least $d_{i_{j}}-1$.

We will prove below that given such a $\tau$, we can always shorten the length of this expression by 2 , and that this expression cannot consist of one $\sigma_{i}$. Hence the only possibility for $\tau$ is to be the identity and $k_{i}=m_{i}$ for all $i$, a contradiction.

We claim that $\epsilon_{1}=1$ and $\epsilon_{s}=-1$. If $\epsilon_{1}$ were -1 , then in order to apply $\sigma_{i_{1}}$ we would need that the exponent $x_{i_{1}}$ in $\omega_{k}$ is at least $d_{i_{1}}-1$, contradicting that $\omega_{k}$ is associated with an admissible monomial type. Similarly, if $\epsilon_{s}=1$ we obtain that the exponent of $x_{i_{s}}$ in $\omega_{m}$ is at least $d_{i_{s}}-1$, contradicting that $\omega_{m}$ is associated with an admissible monomial type.

Let $j$ be the smallest integer such that $\epsilon_{j}=-1$. This implies that the exponent of $x_{i_{j}}$ in $\sigma_{i_{j-1}} \ldots \sigma_{i_{1}}\left(\prod x_{i}^{k_{i}}\right)$ is at least $d_{i_{j}}-1$, hence at least for one of the $j^{\prime}<j$ we have $i_{j}=i_{j^{\prime}}$. Let $j^{\prime}$ be the largest integer smaller than $j$ such that $i_{j}=i_{j^{\prime}}$.

Note that the $\sigma_{i}$ commute as operators on $\mathbb{Q}_{q}\left(x_{0}, \ldots, x_{n}\right)$. Hence, if we consider the $\sigma_{i}$ as operators on $\mathbb{Q}_{q}\left(x_{0}, \ldots x_{n}\right)$ then we have the identities

$$
\begin{aligned}
\sigma_{i_{j}}^{-1} \sigma_{i_{j-1}} \ldots \sigma_{i_{j^{\prime}}} \sigma_{i_{j^{\prime}-1}} \ldots \sigma_{i_{1}}\left(\prod x_{i}^{k_{i}}\right) & =\sigma_{i_{j}}^{-1} \sigma_{i_{j^{\prime}}} \sigma_{i_{j-1}} \ldots \sigma_{i_{j^{\prime}+1}} \sigma_{i_{j^{\prime}-1}} \ldots \sigma_{i_{1}}\left(\prod x_{i}^{k_{i}}\right) \\
& =\sigma_{i_{j-1}} \ldots \sigma_{i_{j^{\prime}+1}} \sigma_{i_{j^{\prime}-1}} \ldots \sigma_{i_{1}}\left(\prod x_{i}^{k_{i}}\right)
\end{aligned}
$$


We need to show that the latter expression corresponds to a series of relations in $P^{t} / P^{t-1}$, i.e., we need to show that for each $j^{\prime \prime}$ such that $j^{\prime}<j^{\prime \prime}<j$, if

$$
\sigma_{i_{j^{\prime \prime}}} \ldots \sigma_{i_{j^{\prime}+1}} \sigma_{i_{j^{\prime}-1}} \ldots \sigma_{i_{1}}\left(\prod x_{i}^{k_{i}}\right)=c \prod x_{r}^{e_{r}}
$$

with $c \in \mathbb{Q}_{q}$ then $e_{r} \geq a_{r}-\delta_{r, i_{j^{\prime \prime}+1}}$ for all $r$.

Suppose that $r \neq i_{j}$. Since

$$
\sigma_{i_{j^{\prime \prime}}} \ldots \sigma_{i_{1}}\left(\prod x_{i}^{k_{i}}\right)=c^{\prime} \prod x_{r}^{e_{r}^{\prime}}
$$

with $c^{\prime} \in \mathbb{Q}_{q}$ and $e_{r}^{\prime} \geq a_{r}-\delta_{r, i_{j^{\prime \prime}+1}}$ and $\sigma_{i_{j}}$ lowers the exponent of $x_{r}$ by $a_{r}$ we obtain $e_{r}^{\prime}=e_{r}-a_{r}$, whence $e_{r} \geq a_{r}-\delta_{r, i_{j^{\prime \prime}+1}}$.

Suppose that $r=i_{j}$. Since

$$
\sigma_{i_{j-1}} \ldots \sigma_{i_{1}}\left(\prod x_{i}^{k_{i}}\right)=c^{\prime \prime} \prod x_{r}^{e_{r}^{\prime \prime}}
$$

with $c^{\prime \prime} \in \mathbb{Q}_{q}$ and $e_{r}^{\prime \prime} \geq d_{r}-1$, it follows that

$$
\sigma_{i_{j-1}} \ldots \sigma_{i_{j^{\prime}+1}} \sigma_{i_{j^{\prime}-1}} \ldots \sigma_{i_{1}}\left(\prod x_{i}^{k_{i}}\right)=c^{\prime \prime \prime} \prod x_{r}^{e_{r}^{\prime \prime \prime}}
$$

with $c^{\prime \prime \prime} \in \mathbb{Q}_{q}$ and $e_{r}^{\prime \prime \prime} \geq 0$. Since the $\sigma_{i_{k}}$ for $j^{\prime \prime}<k<j^{\prime}$ lower the exponent of $x_{r}$ by $a_{r}$ we obtain $e_{r}=e_{r}^{\prime \prime \prime}+\left(j-j^{\prime \prime}\right) a_{r} \geq a_{r}$.

We need to show that

$$
\left\{\omega_{\boldsymbol{k}}: \boldsymbol{k} \text { an admissible monomial type }\right\}
$$

spans $H^{n}\left(U_{\lambda}, \mathbb{Q}_{q}\right)$. If $\lambda=0$ then this follows from the discussion before this proposition. If $\lambda \neq 0$ and all the weights equal 1 then [Katz 1968, Theorem 1.10] shows that $\operatorname{dim} H^{n-1}\left(X_{\lambda}, \mathbb{Q}_{q}\right)$ is independent of $\lambda$. Using (3) we obtain that $\operatorname{dim} H^{n}\left(U_{\lambda}, \mathbb{Q}_{q}\right)$ is independent of $\lambda$. The general case follows from this case by applying the standard quotient map and [Dolgachev 1982, Lemma 2.2.2].

\section{Deformation theory}

Assume for the moment that $\mathbb{P}=\mathbb{P}^{n}$. Following N. Katz, consider the commutative diagram

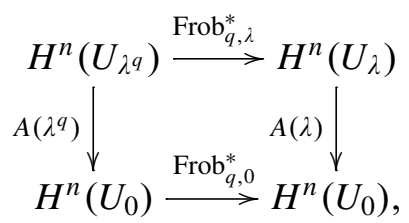

where $\operatorname{Frob}_{q, \lambda}$ is the Frobenius acting on the complete family. Since it maps the fiber over 0 to the fiber over 0 this map can be restricted to $U_{0}$. Katz studied the differential equation associated to $A(\lambda)$. He remarked in a note that $A(\lambda)$ is actually the solution of the Picard-Fuchs equation. 
We first give a way of computing a map $B(\lambda)$ such that

$$
\operatorname{Frob}_{q, 0}^{*} B\left(\lambda^{q}\right)=B(\lambda) \operatorname{Frob}_{q, \lambda}
$$

on a small neighborhood of 0 . This matrix $B(\lambda)$ is enough to deduce Frob ${ }_{q, \lambda}^{*}$ from Frob $_{q, 0}^{*}$.

Fix a basis

$$
\frac{G_{i}}{F_{\lambda}^{t}} \Omega
$$

for $H^{n}\left(U_{\lambda}\right)$ and write

$$
\frac{G_{i}}{F_{\lambda}^{t}} \Omega=\sum_{j=0}^{\infty}\left(\begin{array}{c}
j+t-1 \\
j
\end{array}\right) \frac{G_{i}\left(F-F_{\lambda}\right)^{j}}{F^{j+t}} \Omega .
$$

Since $F-F_{\lambda}$ is the product of $\lambda$ with a polynomial with integral coefficients, the above power series in the $x_{i}$ converges on a small disc. By choosing $\lambda$ sufficiently small, we obtain an overconvergent power series in the $x_{i}$, hence $\frac{G_{i}}{F_{\lambda}^{t}}$ defines an element of $H^{n}\left(U_{0}\right)$. Let $B(\lambda): H^{n}\left(U_{\lambda}\right) \rightarrow H^{n}\left(U_{0}\right)$ be the analytic continuation of the operator mapping

$$
\frac{G_{i}}{F_{\lambda}^{t}} \Omega
$$

to the complete reduction of (4) in $H^{n}\left(U_{0}\right)$.

In this way we obtain a local expansion of the matrix $B(\lambda)$ around $\lambda=0$. In the following section we will make this more explicit.

Proposition 4.1 (Katz). We have $B(\lambda) \operatorname{Frob}_{q, \lambda}^{*}=\operatorname{Frob}_{q, 0}^{*} B\left(\lambda^{q}\right)$ and $B(\lambda)=A(\lambda)$.

Proof. The case $\mathbb{P}=\mathbb{P}^{n}$ is a combination of [Katz 1968, Lemma 2.10, Lemma 2.13, Theorem 2.14]. The general case is a formal consequence of the special case by Lemma 3.7, Proposition 4.1 and the definition of $H^{n}\left(U_{\lambda}, \mathbb{Q}_{q}\right)$ in terms of the standard quotient map $\pi: \mathbb{P}^{n} \rightarrow \mathbb{P}$.

Remark 4.2. Proposition 4.1 is particularly interesting in the case when we specialize to $\lambda=\lambda_{0}$ where $\lambda_{0}$ is the Teichmüller lift of some element $\bar{\lambda}_{0}$. Then $\lambda_{0}^{q}=\lambda_{0}$, hence Frob ${ }_{q, \lambda_{0}}^{*}$ is a lift of Frobenius on $H^{n}\left(U_{\lambda_{0}}, \mathbb{Q}_{q}\right)$. Using Theorem 3.11 and Theorem 3.8 we obtain that

$$
Z\left(U_{\bar{\lambda}_{0}}, t\right)=\lim _{\lambda \rightarrow \lambda_{0}} \frac{\left(\operatorname{det}\left(I-t q^{n} \mid A\left(\lambda^{q}\right)^{-1}\left(\operatorname{Frob}_{q, 0}^{*}\right)^{-1} A(\lambda)\right)\right)^{(-1)^{n+1}}}{1-q^{n} t} .
$$

\section{Actual computation of the deformation matrix}

In order to compute the matrix $A(\lambda)$ we need to reduce the right hand side of (4) in $H^{n}\left(U_{0}\right)$. We start with a very useful lemma. 
Lemma 5.1. Fix nonnegative integers $b_{i}$ such that $\sum b_{i} w_{i}+w=t d$ for some integer $t$. The complete reduction of $\omega:=\frac{\prod x_{i}^{b_{i}}}{F^{t}} \Omega$ equals

$$
\frac{\prod_{i}\left(\left(c_{i}+1\right) w_{i} / d\right)_{q_{i}}}{(s)_{t-s}} \frac{\prod x_{i}^{c_{i}}}{F^{s}} \Omega,
$$

where $0 \leq c_{i}<d_{i}$ and $q_{i}$, s are integers such that $b_{i}=q_{i} d_{i}+c_{i}$, and $s d=\sum c_{i} w_{i}+$ $w$, i.e., $t-s=\sum q_{i}$, provided that $c_{i} \neq d_{i}-1$ for all $i$. If for one of the $i$ we have $c_{i}=d_{i}-1$ then $\omega$ reduces to zero in $H\left(U_{0}, \mathbb{Q}_{q}\right)$.

Proof.

The reduction with respect to $x_{0}$ of

$$
\frac{x_{0}^{b_{0}} \prod_{i=1}^{n} x_{i}^{b_{i}}}{F^{t}} \Omega
$$

(cf. Definition 3.14) equals

$$
\frac{x_{0}^{b_{0}-d_{0}}\left(\left(b_{0}+1\right)-d_{0}\right) \prod x_{i}^{b_{i}}}{(t-1) d_{0} F^{t-1}} \Omega=\frac{x_{0}^{b_{0}-d_{0}}\left(\left(b_{0}+1\right) w_{0}-d\right) \prod x_{i}^{b_{i}}}{(t-1) d F^{t-1}} \Omega
$$

(provided $b_{0} \geq d_{0}$ ). After reducing $q_{i}$ times with respect to $x_{i}$ for $i=0, \ldots, n$, we obtain that $\omega$ reduces to

$$
\frac{(s-1) ! \prod_{i}\left(\prod_{j=0}^{q_{i}-1}\left(\left(c_{i}+1\right) w_{i}+j d\right)\right) \prod x_{i}^{c_{i}}}{(t-1) ! d^{t-s} F^{s}} \Omega .
$$

This in turn equals

$$
\tau:=\frac{(s-1) ! d \sum q_{i} \prod\left(\left(c_{i}+1\right) w_{i} / d\right)_{q_{i}} \prod x_{i}^{c_{i}}}{(t-1) ! d^{t-s} F^{s}} \Omega .
$$

If none of the $c_{i}$ equals $d_{i}-1$ then this is a complete reduction. Using $\sum q_{i}=t-s$ the first formula follows.

If $c_{i}=d_{i}-1$ then we can write $\tau$ as $\left(F_{x_{i}} G / F^{s}\right) \Omega$, where $G$ does not contain the variable $x_{i}$. The reduction of this form is a constant times

$$
\frac{G_{x_{i}}}{F^{s-1}} \Omega \text {. }
$$

Since $G_{x_{i}}=0$, this reduction is zero.

Fix an admissible monomial type $\boldsymbol{k}=\left(w_{0}\left(k_{0}+1\right), \ldots, w_{n}\left(k_{n}+1\right)\right) \in(\mathbb{Z} / d \mathbb{Z})^{n+1}$ of relative degree $t$. We want to calculate the reduction of

$$
\frac{\prod x_{i}^{k_{i}}}{\left(F+\lambda \prod x_{i}^{a_{i}}\right)^{t}} \Omega
$$


in $H^{n}\left(U_{0}\right)$. In order to find a power series expression, we assume that $\lambda$ is sufficiently small, then by (4) this form equals

$$
\frac{\prod x_{i}^{k_{i}}}{F^{t}} \frac{1}{\left(1-\frac{(-\lambda) \prod x_{i}^{a_{i}}}{F}\right)^{t}} \Omega=\sum_{j}\left(\begin{array}{c}
t+j-1 \\
j
\end{array}\right) \frac{\prod x_{i}^{k_{i}+a_{i} j}}{F^{t+j}}(-\lambda)^{j} \Omega .
$$

Note that at most $d$ distinct monomials occur in the reduction of the form.

Definition 5.2. Let $r, s$ be nonnegative integers, let $\alpha_{i} \in \mathbb{Q}_{q}$, for $i \in\{1,2, \ldots, r\}$, let $\beta_{j} \in \mathbb{Q}_{q} \backslash \mathbb{Z}_{<0}$ for $j \in\{1,2, \ldots, s\}$. We define the (generalized) hypergeometric function

$$
{ }_{r} F_{s}\left(\begin{array}{cccc}
\alpha_{1} & \alpha_{2} & \ldots & \alpha_{r} \\
\beta_{1} & \beta_{2} & \ldots & \beta_{s}
\end{array} ; z\right)
$$

to be

$$
\sum_{k=0}^{\infty} b_{j} z^{j}
$$

with $b_{0}=1$, and

$$
\frac{b_{j+1}}{b_{j}}=\frac{\left(j+\alpha_{1}\right) \ldots\left(j+\alpha_{r}\right)}{\left(j+\beta_{1}\right) \ldots\left(j+\beta_{s}\right)(j+1)},
$$

for all positive integers $j$.

Let $d_{i}^{\prime}$ be the order of $a_{i} \bmod d_{i}$ in $\mathbb{Z} / d_{i} \mathbb{Z}$. Let $d^{\prime}$ be the least common multiple of all the $d_{i}^{\prime}$. Set $b_{i}=a_{i} d^{\prime} / d_{i}$. In the following proposition and its proof we identify elements in $a \in \mathbb{Z} / m \mathbb{Z}$ with their representative $\tilde{a} \in \mathbb{Z}$ such that $0 \leq \tilde{a} \leq m-1$.

Proposition 5.3. Let $\boldsymbol{k}$ be an admissible monomial type. Let $t$ be the relative degree of $\boldsymbol{k}$. Write $A(\lambda) \omega_{\boldsymbol{k}}=\sum c_{\boldsymbol{m}}(\lambda) \omega_{\boldsymbol{m}}$, where the sum is taken over all admissible monomial types. Then $c_{\boldsymbol{m}}(\lambda)$ is nonzero only if there is a $j_{0} \in \mathbb{Z}$ with $0 \leq j_{0} \leq d^{\prime}-1$ and such that $\boldsymbol{m}-\boldsymbol{k}=\bar{j}_{0} \boldsymbol{a}$. If this is the case then

$$
\frac{c_{\boldsymbol{m}}(\lambda)}{\operatorname{red} \frac{\prod x_{i}^{a_{i} j_{0}+k_{i}}}{F^{t+j_{0}}} \Omega}
$$

equals

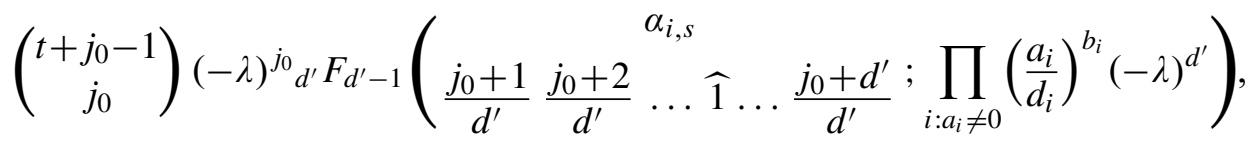

with

$$
\alpha_{i, s}=\frac{(s-1) d_{i}+1+a_{i} j_{0}+k_{i}}{a_{i} d^{\prime}}, s=1, \ldots, b_{i} ; i=0, \ldots n
$$


This proposition almost gives a complete reduction of the form $\operatorname{Frob}_{q, \lambda}\left(\omega_{\boldsymbol{k}}\right)$, in the sense that $c_{k}(\lambda)$ is described as the product of a hypergeometric function and the reduction of a rational function in the $x_{i}$ multiplied by $\Omega$. The latter form can be easily reduced using Lemma 5.1.

Proof. It suffices to compute explicitly a complete reduction of $\omega:=\frac{\prod x_{i}^{k_{i}}}{F_{\lambda}^{t}} \Omega$ in $H^{n}\left(U_{0}\right)$. We can write $\omega$ as

$$
\sum_{j}\left(\begin{array}{c}
t+j-1 \\
j
\end{array}\right) \frac{\prod x_{i}^{k_{i}+a_{i} j}}{F^{t+j}}(-\lambda)^{j} \Omega .
$$

Set $c_{t, j}:=\left(\begin{array}{c}t+j-1 \\ j\end{array}\right)$. Since each reduction step decreases the exponent of $x_{i}$ by $d_{i}$, we split this sum as follows: write

$$
\omega=\sum_{j_{0}=0}^{d^{\prime}-1} \sum_{j} c_{t, j_{0}+d^{\prime} j} \frac{\prod x_{i}^{k_{i}+a_{i}\left(j_{0}+d^{\prime} j\right)}}{F^{t+j_{0}+d^{\prime} j}}(-\lambda)^{j_{0}+d^{\prime} j} \Omega .
$$

For $0 \leq j_{0} \leq d^{\prime}-1$ set

$$
\omega_{j_{0}}:=\sum_{j} c_{t, j_{0}+d^{\prime} j} \frac{\prod x_{i}^{k_{i}+a_{i}\left(j_{0}+d^{\prime} j\right)}}{F^{t+j_{0}+d^{\prime} j}}(-\lambda)^{j_{0}+d^{\prime} j} \Omega .
$$

From Lemma 5.1 it follows that if $k_{i}+a_{i}\left(j_{0}+d^{\prime} j\right) \equiv-1 \bmod d_{i}$ for some $i$, then $\omega_{j_{0}}$ reduces to zero. Otherwise, we claim that the reduction of $\omega_{j}$ is a generalized hypergeometric function. In order to prove this and to calculate the parameters, we need to show that

$$
\frac{c_{t, j_{0}+d^{\prime} j+d^{\prime}} \text { red } \frac{\prod x_{i}^{k_{i}+a_{i}\left(j_{0}+d^{\prime} j\right)+a_{i} d^{\prime}}}{F^{t+j_{0}+d^{\prime} j+d^{\prime}}} \Omega}{c_{t, j_{0}+d^{\prime} j} \operatorname{red} \frac{\prod x_{i}^{k_{i}+a_{i}\left(j_{0}+d^{\prime} j\right)}}{F^{t+j_{0}+d^{\prime} j}} \Omega}
$$

is a rational function in $j$. If we reduce with respect to $x_{i}$ then the exponent of $x_{i}$ is lowered by $d_{i}$. So if we reduce the numerator $b_{i}=a_{i} d^{\prime} / d_{i}$ times with respect to $x_{i}$, then the exponent of $x_{i}$ in the numerator and denominator coincide. Now

$$
\operatorname{red} \frac{\prod x_{i}^{k_{i}+a_{i}\left(j_{0}+d^{\prime} j\right)+a_{i} d^{\prime}}}{F^{t+j_{0}+d^{\prime} j+d^{\prime}}} \Omega
$$

equals

$$
\frac{\prod_{i} \prod_{s=1}^{b_{i}}\left(k_{i}+a_{i}\left(j_{0}+d^{\prime} j\right)+(s-1) d_{i}+1\right)}{\left(t+j_{0}+d^{\prime} j\right)_{\sum b_{i}} \prod_{i} d_{i}^{b_{i}}} \operatorname{red} \frac{\prod x_{i}^{k_{i}+a_{i}\left(j_{0}+d^{\prime} j\right)}}{F^{t+j_{0}+d^{\prime} j}} \Omega
$$


and

$$
\frac{c_{t, j_{0}+d^{\prime} j+d^{\prime}}}{c_{t, j_{0}+d^{\prime} j}}=\frac{\left(t+j_{0}+d^{\prime} j\right)_{d^{\prime}}}{\left(j_{0}+d^{\prime} j+1\right)_{d^{\prime}}} .
$$

Putting this together we conclude that (6) equals

$$
\frac{\prod_{i} \prod_{s=1}^{b_{i}}\left(k_{i}+a_{i}\left(j_{0}+d^{\prime} j\right)+(s-1) d_{i}+1\right)}{\left(j_{0}+d^{\prime} j+1\right) d^{\prime} \prod_{i} d_{i}^{b_{i}}} .
$$

This equals

$$
\frac{\prod_{i: a_{i} \neq 0}\left(a_{i} d^{\prime}\right)^{b_{i}}}{\left(d^{\prime}\right)^{d^{\prime}} \prod_{i: a_{i} \neq 0} d_{i}^{b_{i}}} \frac{\prod_{i} \prod_{s=1}^{b_{i}}\left(j+\frac{(s-1) d_{i}+1+a_{i} j_{0}+k_{i}}{a_{i} d^{\prime}}\right)}{\prod_{s=1}^{d^{\prime}}\left(j+\frac{j_{0}+s}{d^{\prime}}\right)}
$$

Since $\sum b_{i}=d^{\prime}$ the first factor simplifies to

$$
\prod_{i: a_{i} \neq 0}\left(\frac{a_{i}}{d_{i}}\right)^{b_{i}}
$$

From the second factor we can read off the hypergeometric parameters.

Since the first summand of $\omega_{j_{0}}$ equals

$$
(-\lambda)^{j_{0}} c_{t, j_{0}} \frac{\prod x_{i}^{a_{i} j_{0}+k_{i}}}{F^{t+j_{0}}} \Omega,
$$

by collecting everything together, we obtain that

$$
\frac{\operatorname{red} \omega_{j}}{c_{t, j_{0}}(-\lambda)^{j_{0}}}
$$

equals

$$
d^{\prime} F_{d^{\prime}-1}\left(\frac{j_{0}+1}{d^{\prime}} \frac{j_{0}+2}{d^{\prime}} \ldots \widehat{1} \ldots \frac{j_{0}+d^{\prime}}{d^{\prime}} ;(-\lambda)^{d^{\prime}} \prod_{i: a_{i} \neq 0}\left(\frac{a_{i}}{d_{i}}\right)^{b_{i}}\right) \operatorname{red} \frac{\prod x_{i}^{a_{i} j_{0}+k_{i}}}{F^{t+j_{0}}} \Omega,
$$

as desired.

Example 5.4. Consider the family $X^{3}+Y^{3}+Z^{3}+\lambda X Y Z$. Then we obtain the following matrix $A(\lambda)$ (with respect to the basis $\left\{\omega_{(1,1,1)}, \omega_{(2,2,2)}\right\}$ )

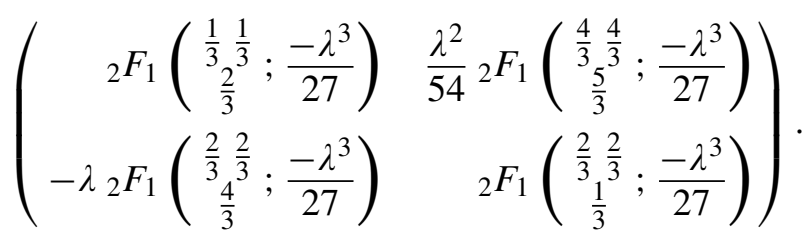


Example 5.5. Another famous example is $C_{\lambda}: X^{4}+Y^{4}+Z^{4}+\lambda X^{2} Y^{2}$.

Note that $d^{\prime}=2, d_{1}^{\prime}=d_{2}^{\prime}=2, d_{3}^{\prime}=0, b_{1}=b_{2}=1, b_{3}=0$.

One easily obtains

$$
\begin{aligned}
& A(\lambda) \omega_{(2,1,1)}={ }_{1} F_{0}\left(\begin{array}{c}
\frac{1}{4} \\
-
\end{array} \frac{\lambda^{2}}{16}\right) \omega_{(2,1,1)}, \quad A(\lambda) \omega_{(1,2,1)}={ }_{1} F_{0}\left(\begin{array}{c}
\frac{1}{4} \\
-
\end{array} \frac{\lambda^{2}}{16}\right) \omega_{(1,2,1)}, \\
& A(\lambda) \omega_{(2,3,3)}={ }_{1} F_{0}\left(\begin{array}{c}
\frac{3}{4} \\
-
\end{array} \frac{\lambda^{2}}{16}\right) \omega_{(2,3,3)}, \quad A(\lambda) \omega_{(3,2,3)}={ }_{1} F_{0}\left(\begin{array}{c}
\frac{3}{4} \\
-
\end{array} \frac{\lambda^{2}}{16}\right) \omega_{(3,2,3)} .
\end{aligned}
$$

$A(\lambda)$ acts as follows on the basis $\left\{\omega_{(1,1,2)}, \omega_{(3,3,2)}\right\}$

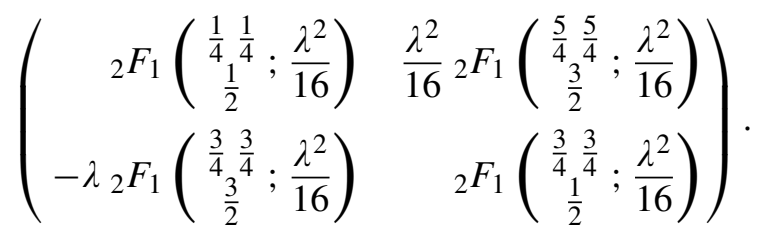

It is classically known that the Jacobian of $C_{\lambda}$ is isogenous to the product of two elliptic curves with $j$-invariant 1728 and one elliptic curve $E_{\lambda}$ whose $j$-invariant depends properly on $\lambda$. This factor can also be obtained from the above information:

When we restrict $A(\lambda)$ to the subspace spanned by $\omega_{(1,1,2),(3,2,2)}$, we find the same operator as the operator $A^{\prime}(\lambda)$ associated with the family $E_{\lambda}: X^{4}+Y^{4}+Z^{2}+$ $\lambda X^{2} Y^{2}$ considered in $\mathbb{P}(1,1,2)$. One easily shows that this is a family of elliptic curves, with $j$-invariant depending on $\lambda$. The curve $E_{0}$ has an automorphism of order 4 with fixed points, hence $j\left(E_{0}\right)=1728$.

In the next section we prove that if $q \equiv 1 \bmod 4$ then all the $\omega_{k}$ are eigenvectors for Frob ${ }_{q, 0}^{*}$, let $c_{\boldsymbol{k}, q}$ be the corresponding eigenvalue. Then, for $\boldsymbol{k}=(2,1,1)$,

$$
\operatorname{Frob}_{q, \lambda}^{*} \omega_{\boldsymbol{k}}=A(\lambda)^{-1} \operatorname{Frob}_{q, 0}^{*} A\left(\lambda^{q}\right) \omega_{\boldsymbol{k}}=\frac{{ }_{1} F_{0}\left(\frac{\frac{1}{4}}{-} ; \frac{\lambda^{2 q}}{16}\right)}{{ }_{1} F_{0}\left(\frac{\frac{1}{4}}{-} ; \frac{\lambda^{2}}{16}\right)} c_{\boldsymbol{k}, q} \omega_{\boldsymbol{k}} .
$$

One easily shows that the factor in front of $c_{k, q}$ is a fourth root of unity, which implies that we have twisted the Frobenius action on $\omega_{k}$ by a quartic character. Something similar happens when $\boldsymbol{k} \in\{(1,2,1),(2,3,3),(3,2,3)\}$. This implies that on a 4-dimensional subspace $V_{\lambda}$ of $H^{1}\left(X_{\lambda}, \mathbb{Q}_{q}\right)$ the Frobenius action is a quartic twist of the Frobenius action on $V_{0} \subset H^{1}\left(X_{0}, \mathbb{Q}_{q}\right)$. The curve $X_{0}$ has the automorphism $[X, Y, Z] \mapsto[Z, X, Y]$. From this we obtain that the action of Frobenius on $V_{0}$ is isomorphic to two copies of the Frobenius action on $E_{0}$.

Example 5.6. Consider now the quintic threefold $X_{0}^{5}+X_{1}^{5}+X_{2}^{5}+X_{3}^{5}+X_{4}^{5}+$ $\lambda X_{0} X_{1} X_{2} X_{3} X_{4}$. This family is studied for example by Candelas, de la Ossa, and 
Rodriguez-Villegas [Candelas et al. 2003]. We discuss another aspect of this family in Example 6.11.

One can distinguish between the following five types of subspaces:

We start with $V_{1}=\operatorname{span}\left\{\omega_{(1,1,1,1,1)}, \omega_{(2,2,2,2,2)}, \omega_{(3,3,3,3,3)}, \omega_{(4,4,4,4,4)}\right\}$. The corresponding matrix is

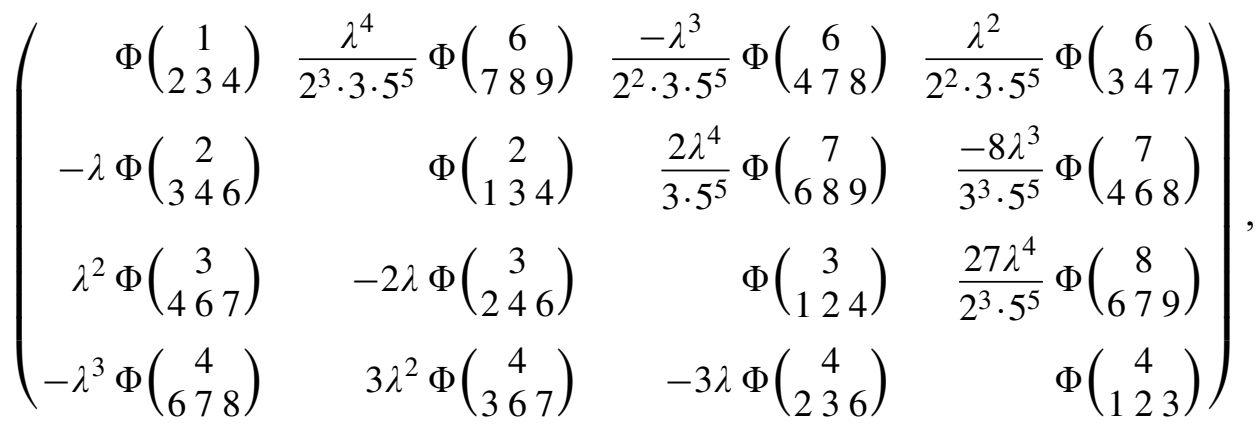

where we used the shorthand

$$
\Phi\left(\begin{array}{c}
a \\
b c d
\end{array}\right):={ }_{4} F_{3}\left(\begin{array}{cccc}
\frac{a}{5} & \frac{a}{5} \frac{a}{5} \frac{a}{5} \\
\frac{b}{5} \frac{c}{5} \frac{d}{5}
\end{array} ; \frac{-\lambda^{5}}{5^{5}}\right) .
$$

The other four spaces are less interesting: on $V_{2}=\operatorname{span}\left\{\omega_{(1,1,1,3,4)}, \omega_{(4,4,4,1,2)}\right\}$, $A(\lambda)$ acts as

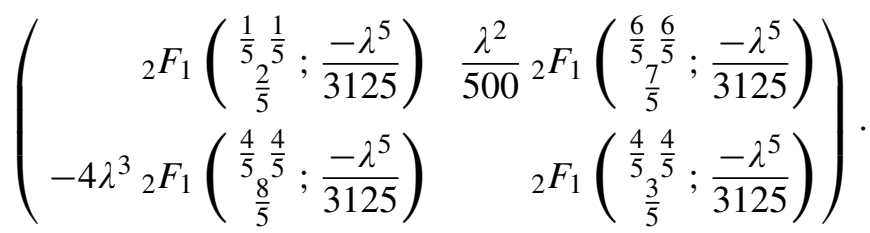

On $V_{3}=\operatorname{span}\left\{\omega_{(2,2,2,1,3)}, \omega_{(3,3,3,2,4)}\right\}, A(\lambda)$ acts as

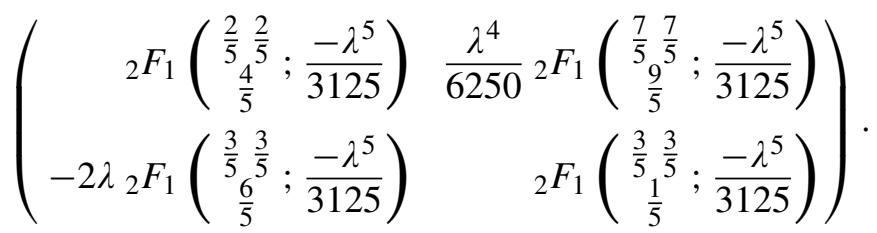

On $V_{4}=\operatorname{span}\left\{\omega_{(1,1,2,2,4)}, \omega_{(3,3,4,4,1)}\right\}, A(\lambda)$ acts as

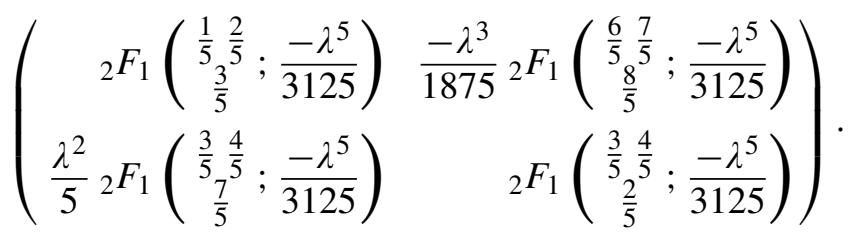


On $V_{5}=\operatorname{span}\left\{\omega_{(3,3,1,1,2)}, \omega_{(4,4,2,2,3)}\right\}, A(\lambda)$ acts as

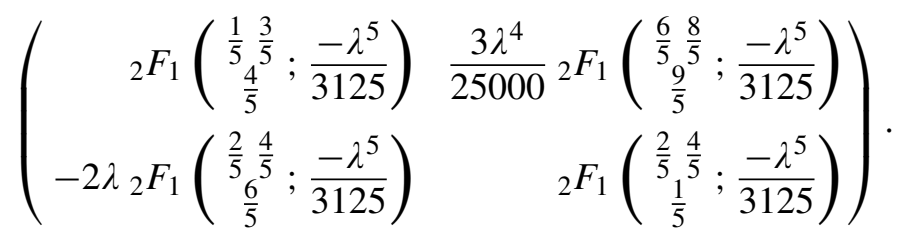

Example 5.7. The final example is the family $X^{4}+Y^{4}+Z^{4}+W^{4}+\lambda X Y Z W$. This is a family of $K 3$-surfaces. This family is also studied in [Dwork 1969, pp. 73-77].

Considered over a number field, every smooth member of this family has geometric Picard number 19 or 20 . This implies that when we consider this family over a finite field, then every smooth member has geometric Picard number at least 20. From the Tate conjecture (which is proven in this case [Nygaard and Ogus 1985] if $p \geq 5$ ) it follows that every smooth member has Picard number 20 or 22. This implies that at least 19 of the eigenvalues of $\operatorname{Frob}_{q, \lambda}^{*}$ on $H^{3}\left(U_{\lambda}\right)$ are of the form $q \zeta$, with $\zeta$ a root of unity. We will indicate how one can obtain this result from the methods described in this section.

First we calculate the operator $A(\lambda)$. We obtain that

$$
A(\lambda) \omega_{(1,2,2,3)}={ }_{1} F_{0}\left(\begin{array}{c}
\frac{1}{2} \\
-
\end{array} \frac{\lambda^{4}}{256}\right) \omega_{(1,2,2,3)} .
$$

The operator $A(\lambda)$ leaves the space spanned by $\omega_{(1,1,3,3)}$ and $\omega_{(3,3,1,1)}$ invariant. Its action is as follows:

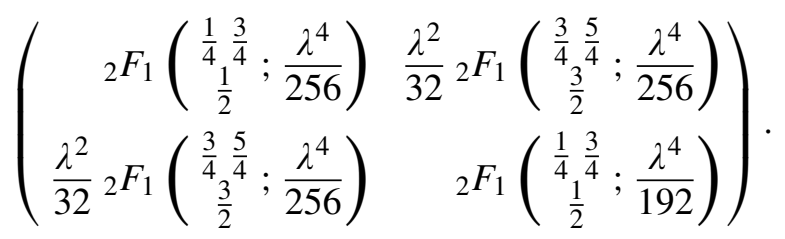

One easily computes that

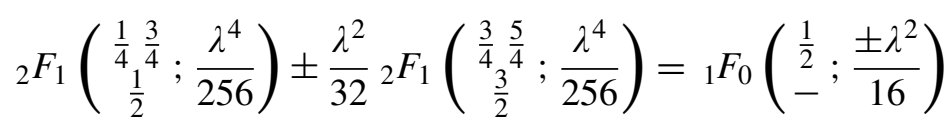

hence

$$
A(\lambda) \omega_{(1,1,3,3)} \pm \omega_{(3,3,1,1)}={ }_{1} F_{0}\left(\frac{\frac{1}{2}}{-} ; \frac{ \pm \lambda^{2}}{16}\right) \omega_{(1,1,3,3)} \pm \omega_{(3,3,1,1)} .
$$

As explained in the previous example, this implies that if $q \equiv 1 \bmod 4$ then Frob ${ }_{\lambda, q}^{*}$ restricted to the subspace generated by the $\omega_{(1,2,2,3)}, \omega_{(1,1,3,3)}$ and all the coordinate permutations of these forms, is a (quartic) twist of $\mathrm{Frob}_{0, q}^{*}$. Using Jacobi sums one can show that the Frob $_{0, q}^{*}$ restricted to this subspace has only eigenvalues of the 
form $q \zeta$, with $\zeta$ a root of unity. This yields 18 eigenvalues of Frob ${ }_{\lambda, q}$ of this form. Since the number of eigenvalues of Frob ${ }_{\lambda, q}^{*}$ that are not of this form is even, and the complementary subspace has dimension 3 , there is a nineteenth eigenvalue of the form $q \zeta$.

The final subspace under consideration is $\operatorname{span}\left\{\omega_{(1,1,1,1)}, \omega_{(2,2,2,2)}, \omega_{(3,3,3,3)}\right\}$. We obtain the following matrix with respect to this basis:

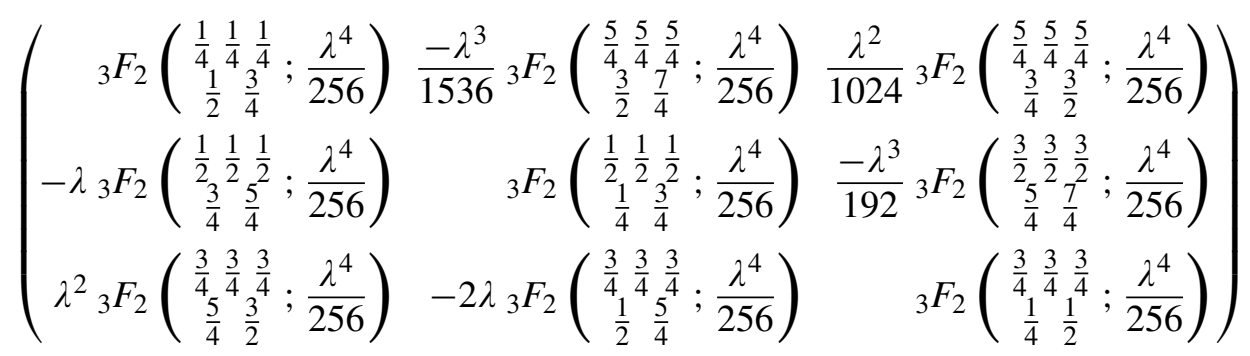

\section{Fermat hypersurfaces and equivalence relations}

In the previous sections it is shown how to calculate the deformation matrix $A(\lambda)$. In this section we discuss the Frobenius action on the central fiber.

Lemma 6.1. Let $\boldsymbol{k}$ be an admissible monomial type. Let $\boldsymbol{m}=\bar{q} \boldsymbol{k}$. We have Frob $_{0, q} \omega_{\boldsymbol{k}}=c_{\boldsymbol{k}, q} \omega_{\boldsymbol{m}}$ for some $c_{\boldsymbol{k}, q}$.

Proof. Take as a lift of Frobenius the morphism $x_{i} \mapsto x_{i}^{q}$. Then

$$
\operatorname{Frob}_{q, 0}^{*}(\omega)=\frac{x_{i}^{q k_{i}+q-1}}{F\left(x_{i}^{q}\right)^{t}} \Omega=\sum_{j=0} c_{t, j} \frac{x_{i}^{q k_{i}}\left(F^{q}-F\left(x_{i}^{q}\right)\right)^{j}}{F^{q j+t}} .
$$

One can easily show that any exponent of $x_{i}$ in this sum is congruent to $q k_{i}+q-$ $1 \bmod d_{i}$. Hence there is only one monomial type $\boldsymbol{m}$ occurring in the reduction, namely $\bar{q} \boldsymbol{k}$.

Remark 6.2. Suppose $q \equiv 1 \bmod d$. It is well-known that the eigenvalues of Frobenius on $H^{n}(U)$ are of the form $q^{n-1} / J_{k, q}$, where $J_{k, q}$ is a so-called Jacobi sum. Note that the assumption on $q$ implies that $\bar{q} \boldsymbol{k}=\boldsymbol{k}$. So the set of Jacobi sums coincides with the set of $c_{k, q}$ (cf. the Introduction). A stronger result will be proved in the sequel.

Definition 6.3. Two monomial types are called strongly equivalent if and only if their difference is a multiple of the deformation vector. Two monomial types are called weakly equivalent if and only if there exists nonzero multiples of both monomial types that differ by the deformation vector.

The characteristic polynomial of Frobenius on the cohomology can be factorized in factors corresponding to the weak-equivalence classes of monomial types: 
Theorem 6.4. Let $\boldsymbol{k}$ be an admissible monomial type. Let $S$ be the set of monomial types that are weakly equivalent to $\boldsymbol{k}$ and let $S^{\prime}$ be the set of monomial types that are strongly equivalent to $\boldsymbol{k}$. Then

$$
\operatorname{Frob}_{\lambda, q} \omega_{\boldsymbol{k}}=\sum_{\boldsymbol{m} \in S} c_{\boldsymbol{m}, q}^{\prime} \omega_{\boldsymbol{m}}
$$

for some $c_{m, q}^{\prime} \in \mathbb{Q}_{q}$. In particular, the characteristic polynomial $P(T)$ of Frobenius on $H^{n}(U)$ can be factored as $P(T)=\prod_{[k]} P_{[k]}(T)$, where the product is taken over all weak-equivalence classes, and $P_{[k]}(T)$ is an element of $\mathbb{Q}_{q}[T]$ of degree equal to the number of distinct admissible monomial types in the weak-equivalence class $[k]$.

If, moreover, $q \equiv 1 \bmod d$ then

$$
\operatorname{Frob}_{\lambda, q} \omega_{\boldsymbol{k}}=\sum_{\boldsymbol{m} \in S^{\prime}} c_{\boldsymbol{m}, q}^{\prime} \omega_{\boldsymbol{m}}
$$

for some $c_{m, q}^{\prime} \in \mathbb{Q}_{q}$. In particular, the characteristic polynomial $P(T)$ of Frobenius on $H^{n}(U)$ can be factored as $P(T)=\prod_{[k]} P_{[k]}(T)$, where the product is taken over all strong-equivalence classes, and $P_{[k]}(T)$ is an element of $\mathbb{Q}_{q}[T]$ of degree equal to the number of distinct admissible monomial types in the strong-equivalence class $[k]$.

Proof. Since $\operatorname{Frob}_{\lambda, q}=A(\lambda)^{-1} \operatorname{Frob}_{\lambda, 0} A\left(\lambda^{q}\right)$, it suffices to prove that all these three operators leave the subspace $\operatorname{span}_{m \in S}\left(\omega_{m}\right)$ (if $q \not \equiv 1 \bmod d$ ) or the subspace $\operatorname{span}_{m \in S^{\prime}}\left(\omega_{m}\right)$ (if $\left.q \equiv 1 \bmod d\right)$ invariant. For $A(\lambda)^{-1}$ and $A\left(\lambda^{q}\right)$ this follows from Proposition 5.3. For Frob ${ }_{\lambda, 0}$ this follows from Lemma 6.1.

Remark 6.5. In Corollary 6.10 we show that the factorization mentioned above gives factors which are polynomials with $\mathbb{Q}$-coefficients rather then with $\mathbb{Q}_{q}$-coefficients.

It remains to show that weak equivalence is the same relation as "indistinguishable by automorphisms".

Definition 6.6. We call $\boldsymbol{b} \in(\mathbb{Z} / d \mathbb{Z})^{n+1}$ an admissible automorphism type if $\boldsymbol{b}$ can be written as $\left(w_{0} b_{0}, w_{1} b_{1}, \ldots, w_{n} b_{n}\right) \in(\mathbb{Z} / d \mathbb{Z})^{n+1}$, such that $\sum w_{i} b_{i} a_{i} \equiv 0 \bmod d$. Define $\sigma_{b}$ to be the automorphism

$$
\left[x_{0}: x_{1}: \cdots: x_{n}\right] \mapsto\left[\zeta_{d}^{w_{0} b_{0}} x_{0}: \zeta_{d}^{w_{1} b_{1}} x_{1}: \cdots: \zeta_{d}^{w_{n} b_{n}} x_{n}\right] .
$$

We call two monomial types $\boldsymbol{k}$ and $\boldsymbol{m}$ distinguishable by automorphisms if there exists an admissible automorphism type $\boldsymbol{b} \in(\mathbb{Z} / d \mathbb{Z})^{n+1}$ such that

$$
\sigma_{\boldsymbol{b}}\left(\prod x_{i}^{k_{i}}\right)=\prod x_{i}^{k_{i}} \quad \text { and } \quad \sigma_{\boldsymbol{b}}\left(\prod x_{i}^{m_{i}}\right) \neq \prod x_{i}^{m_{i}}
$$


Theorem 6.7. Two monomial types $\boldsymbol{k}$ and $\boldsymbol{m}$ are weakly equivalent if and only if $\boldsymbol{k}$ and $\boldsymbol{m}$ are not distinguishable by automorphisms.

Proof. One easily sees that $\sigma_{\left(b_{i}\right)}$ fixes $\omega_{\boldsymbol{k}}$ if and only if $\prod x_{i}^{k_{i}+1}$ is fixed by $\sigma_{\left(b_{i}\right)}$. This in turn is equivalent with

$$
\sum b_{i}\left(k_{i}+1\right) w_{i} \equiv 0 \bmod d,
$$

and similarly for $\boldsymbol{m}$.

(' $\Rightarrow$ '). Suppose $\boldsymbol{k}$ and $\boldsymbol{m}$ are weakly equivalent. Then we have a relation

$$
s \boldsymbol{k}+t \boldsymbol{m}=r \boldsymbol{a},
$$

with $s, t \in(\mathbb{Z} / d \mathbb{Z})^{*}$. It suffices to show that if $\boldsymbol{b}$ is an admissible automorphism type then

$$
\sum b_{i}\left(k_{i}+1\right) w_{i} \equiv 0 \bmod d \Longleftrightarrow \sum b_{i}\left(m_{i}+1\right) w_{i} \equiv 0 \bmod d .
$$

Since $\boldsymbol{k}$ and $\boldsymbol{m}$ are weakly equivalent we have

$$
s \sum b_{i} w_{i}\left(k_{i}+1\right)+t \sum b_{i} w_{i}\left(m_{i}+1\right) \equiv r \sum b_{i} a_{i} w_{i} \equiv 0 \bmod d .
$$

Hence

$$
s \sum b_{i} w_{i}\left(m_{i}+1\right) \equiv-t \sum b_{i} w_{i}\left(k_{i}+1\right) \bmod d .
$$

Since $s$ and $t$ are invertible, the above claim follows.

(' $\Leftarrow$ '). Suppose $\boldsymbol{k}$ and $\boldsymbol{m}$ are not distinguishable by automorphisms. Take $\boldsymbol{b}$ such that $\sigma_{\boldsymbol{b}}\left(\omega_{\boldsymbol{k}}\right)=\omega_{\boldsymbol{k}}$ and $\sigma_{\boldsymbol{b}}\left(\omega_{\boldsymbol{m}}\right) \neq \omega_{\boldsymbol{m}}$ Hence

$$
\sum b_{i}\left(k_{i}+1\right) w_{i} \equiv 0 \bmod d,
$$

and

$$
\sum b_{i}\left(m_{i}+1\right) w_{i} \not \equiv 0 \bmod d
$$

Suppose $\boldsymbol{k}$ and $\boldsymbol{m}$ are weakly equivalent, i.e., we have a relation

$$
s \boldsymbol{k}+t \boldsymbol{m}=r \boldsymbol{a}
$$

where $s$ and $t$ are invertible in $\mathbb{Z} / d \mathbb{Z}$. Then

$$
s \sum b_{i} w_{i}\left(k_{i}+1\right)+t \sum b_{i} q_{i}\left(m_{i}+1\right)-r \sum b_{i} a_{i} q_{i} \equiv 0 \bmod d .
$$

Since the first and third summand are zero, the same holds for the second summand. Contradicting that it should be nonzero. So we cannot have a relation

$$
s \boldsymbol{k}+t \boldsymbol{m}=r \boldsymbol{a} .
$$

Hence $\boldsymbol{k}$ and $\boldsymbol{m}$ are not weakly equivalent. 
Definition 6.8. Assume that $q \equiv 1 \bmod d$ (i.e., $\mathbb{F}_{q} \supset \mathbb{F}_{p}\left(\zeta_{d}\right)$ ). Let $\chi$ be the $d$-th power residue symbol. Let $\boldsymbol{k}$ be an admissible monomial type. Let $\boldsymbol{k}_{i}$ be the $i$-th entry of $\boldsymbol{k}$, i.e., $w_{i}\left(k_{i}+1\right)$. Then the Jacobi-sum associated with $\boldsymbol{k}$ is defined as

$$
J_{k, q}:=(-1)^{n+1} \sum_{\left(v_{1}, \ldots, v_{n}\right) \in \mathbb{F}_{q}^{n}: \sum_{i} v_{i}=-1} \chi\left(v_{1}\right)^{\boldsymbol{k}_{1}} \chi\left(v_{2}\right)^{\boldsymbol{k}_{2}} \ldots \chi\left(v_{n}\right)^{\boldsymbol{k}_{n}} .
$$

Corollary 6.9. Assume $q \equiv 1 \bmod d$. Let $\boldsymbol{k}$ be an admissible monomial type. Let $S$ be the set of monomial types that cannot be distinguished by automorphisms from $\boldsymbol{k}$. Then the sets $S_{1}:=\left\{q^{n} / c_{\boldsymbol{m}, q}: m \in S\right\}$ and $S_{2}:=\left\{J_{\boldsymbol{m}, q}: m \in S\right\}$ coincide.

Proof. Let $G \subset \prod \mathbb{Z} / d_{i} \mathbb{Z}$ be the group of automorphisms that fixes $\omega_{\boldsymbol{k}}$. Then $X_{0} / G$ is a Fermat variety in a different weighted projective space $\mathbb{P}^{\prime}$. It is wellknown that the eigenvalues of Frobenius on the primitive part of $H_{\mathrm{rig}, c}^{n-1}\left(X_{0} / G\right)$ are Jacobi-sums appearing in $S_{2}$; see [Gouvêa and Yui 1995], for example.

The group $H^{n}(U / G)$ is canonically isomorphic with the subspace of $H^{n}(U)$ generated by the forms $\omega_{\boldsymbol{m}}$, where $\boldsymbol{m} \in S$ (this follows from [Dolgachev 1982, Lemma 2.2.2]). This implies that all the $q^{n} / c_{\boldsymbol{m}, q}$ with $\boldsymbol{m} \in S$ are eigenvalues of Frob on $H_{\text {rig }, c}^{n-1}\left(X_{0} / G\right)$. Hence $S_{1}=S_{2}$.

Corollary 6.10. Let $\bar{\lambda} \in \mathbb{F}_{q}$. Let $P(t)$ be the characteristic polynomial of Frob $_{\bar{\lambda}}$ on $H^{n}\left(U_{\lambda}, \mathbb{Q}_{q}\right)$. Then

$$
P(t)=\prod_{[k]} P_{[k]}(t),
$$

where the product is taken over all weak-equivalence classes of admissible monomial types. Let $\boldsymbol{k}$ be an admissible polynomial type. Then $P_{[k]}(t)$ is an element of $\mathbb{Q}[t]$ and its degree equals the number of admissible monomial types that are weakly equivalent with $\boldsymbol{k}$.

Proof. Fix for the moment a monomial type $\boldsymbol{k}$. Let $G_{\boldsymbol{k}} \subset \prod \mathbb{Z} / d_{i} \mathbb{Z}$ be the group of automorphisms that fixes $\omega_{\boldsymbol{k}}$. Then $X_{0} / G_{\boldsymbol{k}}$ is a Fermat variety in a different weighted projective space $\mathbb{P}^{\prime}$ and $H^{n}\left(U_{0} / G_{\boldsymbol{k}}, \mathbb{Q}_{q}\right)$ is canonically isomorphic with the subspace of $H^{n}\left(U_{0}, \mathbb{Q}_{q}\right)$ generated by the form $\omega_{\boldsymbol{m}}$, where $\boldsymbol{m}$ is weakly equivalent with $\boldsymbol{k}$. This enables us to write

$$
H^{n}(U)=\bigoplus_{[k]} H^{n}\left(U / G_{[k]}\right) .
$$

For every weak-equivalence class of monomial types, set $P_{[k]}(t) \in \mathbb{Q}[t]$ to be the characteristic polynomial of Frobenius acting on $H^{n}\left(U / G_{[k]}\right)$. Then $P(t)=$ $\prod P_{[k]}(t)$, we have $P_{[k]}(t) \in \mathbb{Q}[t]$ and

$$
\operatorname{deg}\left(P_{[k]}(t)\right)=\operatorname{dim} H^{n}\left(U / G_{[k]}\right)=\#\{\boldsymbol{m}: \boldsymbol{k} \text { and } \boldsymbol{m} \text { are weakly equivalent }\},
$$

which finishes the proof. 
Example 6.11. Consider the case of the quintic threefold in $\mathbb{P}^{4}$, with deformation vector $a=(1,1,1,1,1)$. Up to interchanging coordinates we have the following five strong equivalence classes:

(1) $[0,0,0,0,0],[1,1,1,1,1],[2,2,2,2,2],[3,3,3,3,3]$.

(2) $[0,0,0,2,3],[3,3,3,0,1]$. (20 Permutations possible)

(3) $[1,1,1,0,2],[2,2,2,1,3]$. (20 Permutations possible)

(4) $[0,0,1,1,3],[2,2,3,3,0]$. (30 Permutations possible)

(5) $[2,2,0,0,1],[3,3,1,1,2]$. (30 Permutations possible)

The classes (2) and (3) form one weak-equivalence class, the same holds for (4) and (5). Over an arbitrary finite field we obtain three distinguishable factors of the zeta function, all three of degree 4 . One factor is occurring with multiplicity 30 , one factor is occurring with multiplicity 20 , and one factor is occurring with multiplicity one. This is in agreement with [Candelas et al. 2003].

\section{Acknowledgements}

The author thanks Klaus Hulek, Shabnam Kadir, Orsola Tommasi, Jaap Top and an anonymous referee for their remarks and suggestions for improvements on a previous version of this paper.

\section{References}

[Baldassarri and Chiarellotto 1994] F. Baldassarri and B. Chiarellotto, "Algebraic versus rigid cohomology with logarithmic coefficients", pp. 11-50 in Barsotti Symposium in Algebraic Geometry (Abano Terme, 1991), Perspect. Math. 15, Academic Press, San Diego, CA, 1994. MR 96f:14024 Zbl 0833.14010

[Berthelot 1983] P. Berthelot, "Géométrie rigide et cohomologie des variétés algébriques de caractéristique p", exposé J2 in Study group on ultrametric analysis (Marseille, 1982), Inst. Henri Poincaré, Paris, 1983. MR 85j:14036 Zbl 0515.14015

[Berthelot 1997a] P. Berthelot, "Dualité de Poincaré et formule de Künneth en cohomologie rigide", C. R. Acad. Sci. Paris Sér. I Math. 325:5 (1997), 493-498. MR 2000c:14023 Zbl 0908.14006

[Berthelot 1997b] P. Berthelot, "Finitude et pureté cohomologique en cohomologie rigide", Invent. Math. 128:2 (1997), 329-377. MR 98j:14023 Zbl 0908.14005

[Candelas et al. 2003] P. Candelas, X. de la Ossa, and F. Rodriguez-Villegas, "Calabi-Yau manifolds over finite fields, II", pp. 121-157 in Calabi-Yau varieties and mirror symmetry (Toronto, 2001), edited by N. Yui and J. D. Lewis, Fields Inst. Commun. 38, Amer. Math. Soc., Providence, RI, 2003. MR 2004m:11095 Zbl 1100.14032

[Dolgachev 1982] I. Dolgachev, "Weighted projective varieties", pp. 34-71 in Group actions and vector fields (Vancouver, 1981), edited by J. B. Carrell, Lecture Notes in Math. 956, Springer, Berlin, 1982. MR 85g:14060 Zbl 0516.14014

[Dwork 1969] B. Dwork, “p-adic cycles”, Inst. Hautes Études Sci. Publ. Math. 37 (1969), 27-115. MR 45 \#3415 Zbl 0284.14008 
[Étesse and Le Stum 1993] J.-Y. Étesse and B. Le Stum, "Fonctions $L$ associées aux $F$-isocristaux surconvergents, I: Interprétation cohomologique”, Math. Ann. 296 (1993), 557-576. MR 94i:14030 Zbl 0789.14015

[Gouvêa and Yui 1995] F. Q. Gouvêa and N. Yui, Arithmetic of diagonal hypersurfaces over finite fields, London Math. Soc. Lecture Note Series 209, Cambridge University Press, Cambridge, 1995. MR 97k:11095 Zbl 0833.14015

[Hulek and Kloosterman 2007] K. Hulek and R. Kloosterman, "The $L$-series of a cubic fourfold", Manuscripta Math. 124:3 (2007), 391-407.

[Kadir 2004] S. N. Kadir, The arithmetic of Calabi-Yau manifolds and mirror symmetry, Ph.D. thesis, Oxford University, 2004.

[Kadir and Yui 2006] S. N. Kadir and N. Yui, "Fermat motives and mirror-symmetry for Calabi-Yau hypersurfaces", preprint, 2006. math/0606707

[Katz 1968] N. M. Katz, "On the differential equations satisfied by period matrices", Inst. Hautes Études Sci. Publ. Math. 35 (1968), 223-258. MR 39 \#4168 Zbl 0159.22502

[Katz 2007] N. M. Katz, "Another look at the Dwork family", preprint, 2007, Available at http:// www. math.princeton.edu/ nmk/dworkfam64.pdf.

[Lauder 2004] A. G. B. Lauder, "Counting solutions to equations in many variables over finite fields", Found. Comput. Math. 4:3 (2004), 221-267. MR 2005f:14048 Zbl 1076.11040

[Nygaard and Ogus 1985] N. Nygaard and A. Ogus, "Tate's conjecture for $K 3$ surfaces of finite height”, Ann. of Math. (2) 122:3 (1985), 461-507. MR 87h:14014 Zbl 0591.14005

[Rojas-Leon and Wan 2007] A. Rojas-Leon and D. Wan, "Moment zeta functions for Toric CalabiYau hypersurfaces", preprint, 2007. math/0702679

[Shioda and Inose 1977] T. Shioda and H. Inose, "On singular K3 surfaces", pp. 119-136 in Complex analysis and algebraic geometry: A collection dedicated to K. Kodaira, edited by W. L. Baily, Jr. and T. Shioda, Iwanami Shoten, Tokyo, 1977. MR 56 \#371 Zbl 0374.14006

[Steenbrink 1977] J. Steenbrink, "Intersection form for quasi-homogeneous singularities", Compositio Math. 34:2 (1977), 211-223. MR 56 \#11995 Zbl 0347.14001

Communicated by Hélène Esnault

Received 2007-03-05 Revised 2007-08-31 Accepted 2007-10-08

kloosterman@math.uni-hannover.de

Institut für Algebraische Geometrie, Leibniz Universität Hannover, Welfengarten 1, D-30167 Hannover, Germany 


\section{Algebra \& Number Theory}

www.jant.org

\section{EDITORS}

\section{MANAGING EDITOR}

Bjorn Poonen

University of California

Berkeley, USA

\author{
EDITORIAL BOARD CHAIR \\ David Eisenbud \\ University of California \\ Berkeley, USA
}

\section{BOARD OF EDITORS}

Georgia Benkart

Dave Benson

Richard E. Borcherds

John H. Coates

J-L. Colliot-Thélène

Brian D. Conrad

Hélène Esnault

Hubert Flenner

Edward Frenkel

Andrew Granville

Joseph Gubeladze

Ehud Hrushovski

Craig Huneke

Mikhail Kapranov

Yujiro Kawamata

János Kollár

Hendrik W. Lenstra

Yuri Manin

Barry Mazur
University of Wisconsin, Madison, USA

University of Aberdeen, Scotland

University of California, Berkeley, USA

University of Cambridge, UK

CNRS, Université Paris-Sud, France

University of Michigan, USA

Universität Duisburg-Essen, Germany

Ruhr-Universität, Germany

University of California, Berkeley, USA

Université de Montréal, Canada

San Francisco State University, USA

Hebrew University, Israel

University of Kansas, USA

Yale University, USA

University of Tokyo, Japan

Princeton University, USA

Universiteit Leiden, The Netherlands

Northwestern University, USA

Harvard University, USA
Susan Montgomery

Shigefumi Mori

Andrei Okounkov

Raman Parimala

Victor Reiner

Karl Rubin

Peter Sarnak

Michael Singer

Ronald Solomon

Vasudevan Srinivas

J. Toby Stafford

Bernd Sturmfels

Richard Taylor

Ravi Vakil

Michel van den Bergh

Marie-France Vignéras

Kei-Ichi Watanabe

Andrei Zelevinsky

Efim Zelmanov
University of Southern California, USA

RIMS, Kyoto University, Japan

Princeton University, USA

Emory University, USA

University of Minnesota, USA

University of California, Irvine, USA

Princeton University, USA

North Carolina State University, USA

Ohio State University, USA

Tata Inst. of Fund. Research, India

University of Michigan, USA

University of California, Berkeley, USA

Harvard University, USA

Stanford University, USA

Hasselt University, Belgium

Université Paris VII, France

Nihon University, Japan

Northeastern University, USA

University of California, San Diego, USA

\section{PRODUCTION}

ant@mathscipub.org

Paulo Ney de Souza, Production Manager

Silvio Levy, Senior Production Editor

See inside back cover or www.jant.org for submission instructions.

The subscription price for 2007 is US \$120/year for the electronic version, and \$180/year for print and electronic. Subscriptions, requests for back issues from the last three years and changes of subscribers address should be sent to Mathematical Sciences Publishers, Department of Mathematics, University of California, Berkeley, CA 94720-3840, USA.

Algebra \& Number Theory (ISSN 1937-0652) at Mathematical Sciences Publishers, Department of Mathematics, University of California, Berkeley, CA 94720-3840 is published continuously online. Periodical rate postage paid at Berkeley, CA 94704, and additional mailing offices.

ANT peer-review and production is managed by EditFLOW ${ }^{\mathrm{TM}}$ from Mathematical Sciences Publishers.

PUBLISHED BY

mathematical sciences publishers

http://www.mathscipub.org

A NON-PROFIT CORPORATION

Typeset in LATEX

Copyright $@ 2007$ by Mathematical Sciences Publishers 


\section{Algebra \& Number Theory}

Volume $1 \quad$ No. $4 \quad 2007$

Finite descent obstructions and rational points on curves

MiCHAEL STOLL

Del Pezzo surfaces and representation theory

Vera V. Serganova and Alexei N. Skorobogatov

The zeta function of monomial deformations of Fermat hypersurfaces

REMKE KLOOSTERMAN

THOMAS F. LAM and MARK SHIMOZONO 